\title{
Composición faunística y fenología de las mariposas (Rhopalocera: Papilionoidea) de Cañón de Lobos, Yautepec, Morelos, México
}

\author{
Faunistic composition and phenology of butterflies (Rhopalocera: Papilionoidea) at Cañón de \\ Lobos, Yautepec, Morelos, Mexico
}

\author{
María de las Mercedes Luna-Reyes ${ }^{1}$, Jorge Llorente-Bousquets ${ }^{2 *}$, Armando Luis-Martínez ${ }^{2}$ e Isabel Vargas- \\ Fernández ${ }^{2}$ \\ ${ }^{I}$ Museo de Zoología. Facultad de Estudios Superiores Zaragoza, Universidad Nacional Autónoma de México. Batalla 5 de Mayo s/n. Col. Ejército de \\ Oriente, Iztapalapa, 09230 México D.F., México. \\ ${ }^{2}$ Museo de Zoología "Alfonso L. Herrera", Facultad de Ciencias, Universidad Nacional Autónoma de México. Apartado postal 70-399, 04510 \\ México D.F., México. \\ *Correspondencia: jlb@hp.fciencias.unam.mx
}

\begin{abstract}
Resumen. Con el propósito de conocer las especies de Papilionoidea presentes en Cañón de Lobos, Yautepec, Morelos y describir su distribución temporal, se efectuaron recolectas mensuales desde agosto de 1983 hasta diciembre de 1986. Se reconocieron 145 especies de 4 familias de Papilionoidea que sumadas a los registros obtenidos de la literatura hacen un total de 148 especies para el área; 21 de las cuales son endémicas de México. Nymphalidae y Lycaenidae fueron las familias de mayor riqueza; Nymphalidae fue la más abundante. Como en otras áreas tropicales, la distribución temporal de los Papilionoidea está estrechamente asociada con la precipitación, por lo que hubo más especies y ejemplares durante la temporada lluviosa. Se encontró que la mayor parte de la ropalocerofauna de Cañón de Lobos está conformada por especies escasas y raras residentes en el área; sólo el $10 \%$ del total está asociada exclusivamente a la selva baja caducifolia. En comparación con otras cañadas de Morelos, Cañón de Lobos tiene una de las mayores riquezas de la entidad.
\end{abstract}

Palabras clave: abundancia, estacionalidad, mariposas diurnas, riqueza, selva baja caducifolia.

\begin{abstract}
In order to know the species of Papilionoidea in the area and to describe its temporary distribution, the specimens were monthly captured from August of 1983 to December of 1986. 145 species of 4 families of Papilionoidea were recognized, and added to the obtained registries of literature with a total of 148 species for Cañón de Lobos; 21 of them are endemic to Mexico. Nymphalidae and Lycaenidae were the richest families, while Nymphalidae was the most abundant. Like in other tropical areas, the temporary distribution of the Papilionoidea is associated closely with the precipitation, reason why there were more species and specimens during the rainy season. The most of rhopalocerofauna of Cañón de Lobos is conformed by scarse and rare species that are resident in the area; only $10 \%$ of the species is associated exclusively to the dry forest. In comparison with other localities of Morelos, Cañón de Lobos has one of the greater richness.
\end{abstract}

Key words: abundance, diurnal butterflies, dry forest, richness, seasonality.

\section{Introducción}

Este trabajo es el primero de una serie de investigaciones que integran un proyecto general a largo plazo intitulado "Lepidopterofauna de la selva baja caducifolia", que se desarrolla en el Museo de Zoología de la Facultad de Estudios Superiores Zaragoza, UNAM (MZFESZ), con el cual se pretende iniciar el estudio formal de las mariposas asociadas con este tipo de vegetación y al mismo tiempo conformar una colección sistemática especializada de las especies de este tipo de selva, que a la fecha no existe en

Recibido: 02 junio 2009; aceptado: 29 septiembre 2009 nuestro país.

Generalmente, los trabajos sobre mariposas se circunscriben a fronteras políticas y muy pocos a una unidad biótica o fisiográfica, por lo cual muchas comunidades de mariposas aún no se conocen bien, como ocurre con las que se encuentran asociadas a los ambientes extremos, húmedos o secos (Luis y Llorente, 1990).

Se sabe poco sobre la entomofauna y en especial sobre las mariposas de los ambientes xéricos de México, pues tales estudios básicamente han tratado de reconocer la composición florística y sus relaciones. Uno de los tipos de vegetación que se desarrolla en estas formaciones es la selva baja caducifolia (SBC), también conocida como 
bosque tropical caducifolio, en el que existe gran riqueza florística, con porcentajes altos de endemismo concentrados principalmente en la cuenca del río Balsas, en la península de Yucatán y al noreste de México (Rzedowski, 1978, 1992); también se caracteriza por diversidad alta de vertebrados (Flores y Gerez, 1994).

La SBC es la vegetación tropical con mayor distribución de México (Trejo, 1996), en especial a lo largo de la vertiente del Pacífico, desde Sonora hasta Chiapas, particularmente en las áreas de altitud menor hasta el litoral, además de manchones aislados en la región meridional de Baja California Sur. En la vertiente oriental se registra en porciones relativamente disyuntas de los estados de Tamaulipas, San Luis Potosí, Querétaro, Veracruz, Campeche y Yucatán, mientras que en el centro del país se distribuye en regiones bien delimitadas de Puebla, Morelos y Estado de México. Se desarrolla desde el nivel del mar hasta los $1900 \mathrm{~m}$ de altitud, por lo general debajo de la cota de los $1500 \mathrm{~m}$, aunque en algunos lugares no se establece más allá de los $800 \mathrm{~m}$, como ocurre en zonas del golfo de México. Algunos de los factores más importantes que determinan su distribución geográfica son la temperatura y la humedad, pues existen 2 estaciones bien definidas: la de sequía y la de lluvias; en general, la primera ocurre entre los meses de diciembre y mayo, produciendo en la vegetación una fisonomía muy particular (Rzedowski, 1978).

En 1992, Flores y Gerez (1994) estimaron que la SBC cubría menos del $7 \%$ de la superficie total del país, valor que actualmente puede ser menor si se considera que este dato prácticamente representa la mitad del área que ocupaba en 1980; en el caso del estado de Morelos, más de la mitad de su superficie $(61.38 \%)$ se destina a usos agropecuarios y una fracción pequeña (6.98\%) está ocupada por vegetación natural de SBC y bosques templados.

En particular, sobre las mariposas del estado de Morelos se conocen algunos trabajos desarrollados en áreas de SBC (Medellín, 1985; Maza et al., 1995a, 1995b; Silva e Ibarra, 2003; Luna-Reyes, 2007a; Luna-Reyes et al., 2008), y otros realizados en tipos de vegetación distintos (Maza, 1975; Lara, 1999; Valencia, 1999; LunaReyes y Llorente, 2004; Vences, 2004; Luna-Reyes, 2007b); adicionalmente, la entidad ha sido considerada en estudios de riqueza comparativa y sobre distribución de los papilionoideos mexicanos (Llorente et al., 1996, 1997, 2006; Luis et al., 2003a).

El propósito fundamental de este estudio es conocer la composición faunística de los Papilionoidea de Cañón de Lobos, analizar su abundancia y su variación mensual, así como describir la distribución estacional y el grado de asociación ecológica de la ropalocerofauna. Cañón de Lobos muestra gran variedad de microhábitats y, por consiguiente, una fauna muy diversa, es de fácil acceso y cercano al Distrito Federal. Aunque se conocen estudios faunísticos de áreas próximas (Tepoztlán y Rancho Viejo, Morelos), su fauna presenta diferencias por sus particularidades fisiográficas.

\section{Materiales y métodos}

Área de estudio. Se localiza al SE de Cuernavaca, desde del kilómetro 17 al 20 de la carretera 138 que comunica esta ciudad con la de Cuautla, y entre las coordenadas $18^{0}$ 51'-18 $52^{\circ}$ ' N y 990 06'- 990 08' O (Fig. 1); políticamente pertenece al municipio de Yautepec y está muy próximo al poblado de Amador Salazar.

Cañón de Lobos se encuentra en una cañada formada entre 2 elevaciones de una pequeña cordillera que se deriva de la sierra del Ajusco y se denomina sierra de Yautepec; divide los valles de Cuernavaca y Yautepec en dirección norte-sur (Vidal, 1980), con altitudes que van desde los 1200 a los $1840 \mathrm{~m}$. Fisiográficamente, esta localidad corresponde a una Sierra de Laderas Escarpadas de la subprovincia de Sierras y Valles Guerrerenses de la provincia Sierra Madre del Sur (SPP, 1981). Desde el punto de vista hidrológico pertenece a la región denominada Río Balsas que ocupa la mayor parte del estado de Morelos (SPP, 1981). Durante los meses lluviosos se forma una corriente intermitente de casi $6 \mathrm{~km}$ de longitud a todo lo largo del cañón y paralelo a la carretera. El clima de la zona es Aw ${ }_{0}$ ', (w)(i')g, es decir un cálido subhúmedo, el más seco de los subhúmedos, con régimen de lluvias de verano y presencia de canícula, con un porcentaje de lluvias invernal menor del 5\%; con poca oscilación térmica y marcha anual de la temperatura tipo Ganges (Luna-Reyes, 2007a). La vegetación en Cañón de Lobos es SBC, en la que los árboles más altos tienen una altura promedio menor de $15 \mathrm{~m}$, los cuales pierden casi todas las hojas en la época seca; por lo general son árboles sin espinas que tienen abundantes bejucos (Miranda y Hernández X., 1963). Aunque puede encontrarse en gran variedad de condiciones topográficas y tipos de suelo, con preferencia se halla en suelos someros y pedregosos o en las laderas de las elevaciones montañosas. En este tipo de vegetación, la mayoría de las especies presentan afinidad neotropical y muy pocas o ninguna por la holártica (Rzedowski, 1978).

Es frecuente que haya un estrato arbóreo y uno arbustivo, este último varía en composición de un lugar a otro. Cuando hay poca perturbación, el estrato herbáceo está poco desarrollado o falta casi por completo; existen pocas trepadoras y epífitas, encontrándose preferentemente en las cañadas (Rzedowski, 1978).

Las especies arbóreas presentes en Cañón de Lobos 


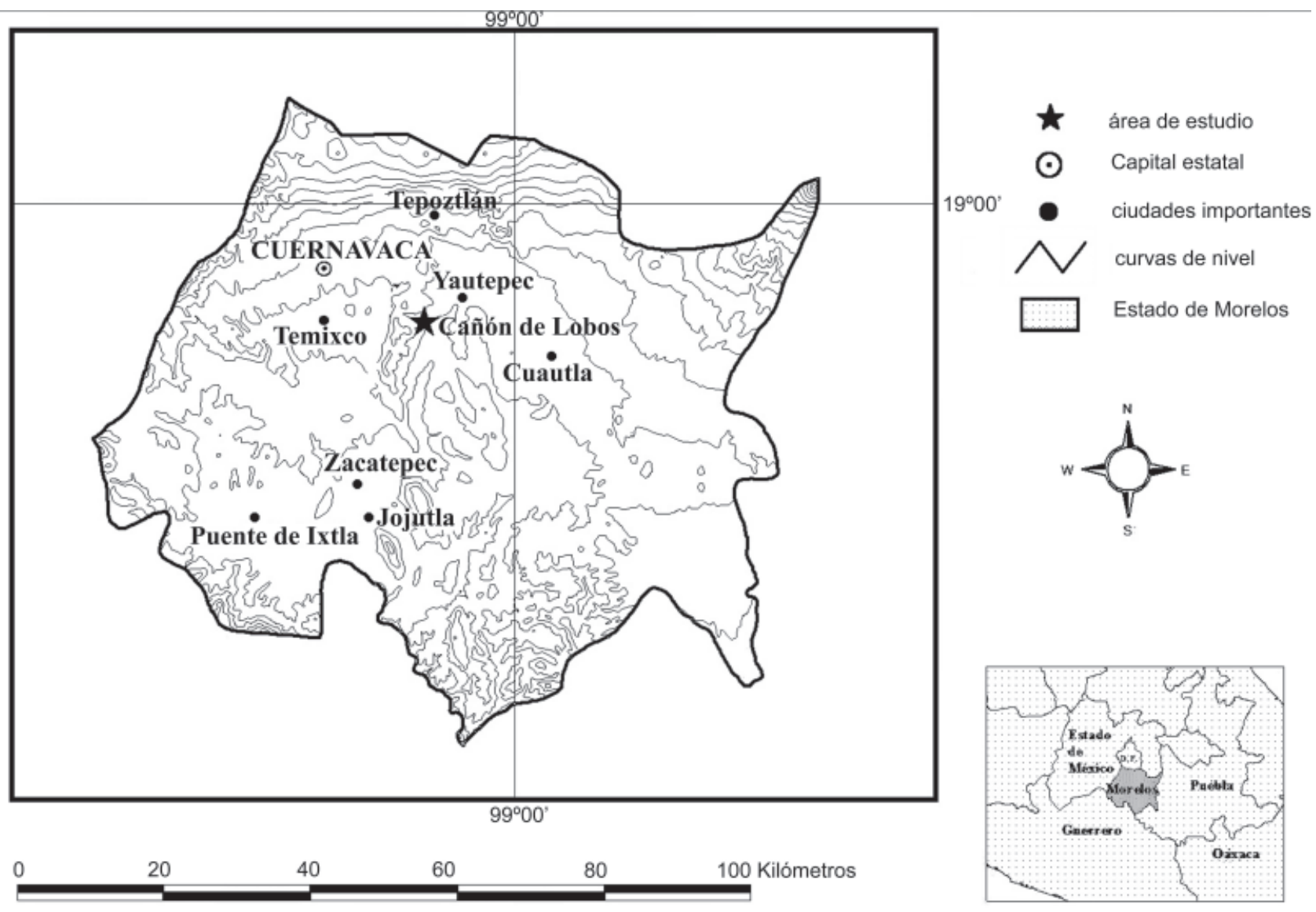

Figura 1. Ubicación del área de estudio en el estado de Morelos, México.

son: Ceiba aesculifolia, Bursera schlechtendalii, Pseudobombax ellipticum, Sapium macrocarpum, Piscidia grandiflora var. glabrescens, Cedrela oaxacensis, Bursera fagaroides, Bursera aff. simaruba, Acacia pennatula, Ipomoea arborea, Plumeria acutifolia, Mastichodendron capiri var. tempisque, Ficus cotinifolia, Comocladia engleriana, Bursera longipes, Hauya elegans, Cordia sp., Coutarea latiflora, Amphipterigium adstringens, Jacquinia aurantiaca, Guazuma ulmifolia, Trichilia pringlei, Lonchocarpus rugosus, Actinochaetia filicina, Erythrina blabelliformis, Vitex mollis y Sickingia sp. Entre las especies arbustivas se encuentran Mimosa biuncifera, Coubrina greggi, Physodium dubium, Hippocratea acapulcensis, Diphysa racemosa vel. aff., Ptelea trifoliata, Karwinskia humboldtiana, Phyllantus sp. y Colubrina macrocarpa; entre las trepadoras Machaerium spp., Amphylophylum paniculatum, Dioscorea subtomentosa y Dioscorea sp., y entre las herbáceas Mirabilis xalapa, Cuphea sp. y Dorstenia drakena. En los lugares con mayor perturbación se presenta Brahea dulcis e Ipomoea arborea.
Recolecta y muestreo de Papilionoidea. Se efectuaron 28 recolectas mensuales de un día de duración en las que participaron 2 personas, de agosto de 1983 hasta diciembre de 1986. A partir de octubre de 1983 se inició la construcción de la carretera de doble carril que comunica las ciudades de Cuernavaca y Cuautla, por lo que en diciembre de 1983 y mayo de 1984 no se permitió el acceso a la zona de estudio y no se tienen estos datos. Afortunadamente, con frecuencia esos meses son de gran escasez poblacional y de riqueza reducida en cuanto a imagos (Vargas et al., 1992; Luna-Reyes et al., 2008). De diciembre de 1984 a septiembre de 1985 no se practicaron recolecciones debido a problemas logísticos y de seguridad que se presentaron en el área; después de este periodo se reanudó el trabajo de campo de manera ininterrumpida durante 14 meses más.

Todos los ejemplares se capturaron con redes entomológicas aéreas durante las horas de mayor actividad de los imagos, esto es, entre las 0900 y las 1600 horas (Howe, 1975; Moore, 1975; Pollard, 1977; Clench, 1979; Brown, 1979). En cada periodo de trabajo se realizó un 
recorrido a lo largo del lecho de un arroyo (utilizándolo como transecto 'lineal'), que en ese punto corre paralelo a la carretera y es la parte más profunda del cañón. La zona de estudio presenta áreas abiertas con gran cantidad de inflorescencias, además de que la disposición de la vegetación no obstaculizó la captura de los ejemplares. Sólo algunos tramos del lecho del río estaban cubiertos casi completamente por árboles, por lo que, a pesar de que no se observó una corriente continua, en la época de lluvias el agua se evaporaba con lentitud formándose charcos o zonas de suelo húmedo, donde se capturaron algunas especies de piéridos y licénidos; sin embargo, la mayoría de los ejemplares fueron capturados al vuelo.

En la determinación taxonómica de los ejemplares se utilizó literatura especializada y se consultaron las colecciones lepidopterológicas del Museo de Zoología de la Facultad de Ciencias (MZFC) y del MZFESZ, ambos de la UNAM, que se tomaron como referencia. El material fue preparado y rotulado de acuerdo con las técnicas convencionales (Howe, 1975), y depositado en la colección lepidopterológica del MZFESZ. Con la información taxonómica y los datos de campo se elaboró el registro escrito, y posteriormente el registro electrónico (Excel); a partir de éste se integró la lista de especies de los papilionoideos de Cañón de Lobos y se analizaron los datos. La representatividad de la riqueza de especies encontrada fue evaluada al elaborar la curva de acumulación de especies y aplicando la fórmula de Clench (1979), que es la que se ajusta mejor para este tipo de estudios (Soberón y Llorente, 1993; Moreno y Halffter, 2001; Jiménez-Valverde y Hortal, 2003).

Como complemento al inventario de especies obtenido en este trabajo se integró una lista de los papilionoideos que habitan el área con la consulta de todos los números de las revistas Bulletin Allyn Museum, Journal of the Lepidopterists' Society, Revista de la Sociedad Mexicana de Lepidopterología, The Journal of Research on the Lepidoptera, Anales del Instituto de Biología y Folia Entomológica Mexicana, que incluyó revisiones de los géneros Panthiades y Cycnus (Eumaeini), Hamadryas, Epiphile, Nessaea, Calephelis, Myscelia, Catonephele y algunos géneros de Euptychiini (Satyrinae). Con la misma finalidad se revisaron las obras Biologia CentraliAmericana (Godman y Salvin, 1869-1901), Die GrossSchmetterlinge der Erde (Seitz, 1912-1924) y el Catálogo sistemático y zoogeográfico de los lepidópteros mexicanos (Hoffmann, 1940).

Las especies fueron agrupadas en 5 categorías de acuerdo con su abundancia total siguiendo la escala geométrica en agrupamientos de tamaño por 4: R (rara: especies con un ejemplar), E (escasa: de 2 a 5), F (frecuente: de 6 a 21), C (común: desde 22 hasta 81), y A (abundante: con 82 ejemplares o más) (Lamas, 1984; Krebs, 1985; Luis y Llorente, 1990 y Luna-Reyes y Llorente, 2004).

En el análisis de la distribución temporal de los papilionoideos, los datos fueron agrupados por mes $\mathrm{y}$ después por estación (primavera: marzo- mayo; verano: junio-agosto; otoño: septiembre-noviembre; invierno: diciembre-febrero); en este caso se tomaron en cuenta los datos de la abundancia estándar (número de ejemplares/ esfuerzo de captura). Con los datos agrupados se describió la riqueza y abundancia por familia, así como la variación poblacional de algunas de las especies más abundantes para correlacionarla con la información climatológica. En esta parte del análisis se tomaron como referencia los datos de Ticumán, que es la estación meteorológica más próxima y está ubicada a una altitud similar, con los mismos tipos de clima y de vegetación que Cañón de Lobos (Fig. 2).

Finalmente, en la evaluación del grado de asociación ecológica de los Papilionoidea de Cañón de Lobos, primero se definieron las especies que pudieran considerarse como residentes del área de estudio; entonces, se elaboró la lista de las plantas de alimentación larval de las especies registradas, con base en los trabajos de Klots (1951), Comstock y Vázquez (1961), Ehrlich y Ehrlich (1961), Vázquez y Pérez (1962), Kendall (1964, 1976), Ross (1964a, 1964b), Ehrlich y Raven (1965), Emmel et al. (1970), Masters (1970), McAlpine (1971), Serrano y Serrano (1972), Tietz (1972), Emmel y Emmel (1973), Neck (1973), Shapiro (1974), Howe (1975), Maza (1975), Riley (1975), Tyler (1975), Scriber y Feeny (1976), Beutelspacher (1980, 1984), Pyle y Knopf (1981), Kendall y McGuire (1984), Lamas (1984), DeVries (1985, 1987, 1997), Scott (1986), Maza (1987), Miller y Miller (1988), y en la información del Índice de plantas huésped para mariposas (FURN, 2006).

La lista florística para Cañón de Lobos se integró con base en los trabajos botánicos de Chavelas (1972), Vázquez (1974) y Soria (1986); además se obtuvieron datos florísticos del estado de Morelos (Corona, 1967; SPP, 1981; Rzedowski y Rzedowski, 2001). La información sobre los huéspedes de Papilionoidea fue contrastada con la información botánica del área de estudio y la de Morelos; así se obtuvo la relación de las plantas de alimentación potenciales de las larvas de los papilionoideos capturados. También, se reconocieron las especies que están asociadas particularmente a la SBC, para lo cual se comparó la lista de Cañón de Lobos con aquellas citadas de otras localidades cuyas características vegetacionales son similares, tales como Rancho Viejo (Maza, 1975) y Cañada Acueducto, Mor. (Maza et al., 1995); Chiautla, Pue. (Luna-Reyes et al., 2008); Chorros del Varal (Arteaga, 1991) y Pedernales, Mich. (Balcázar, 1993); Chamela, Jal. (Beutelspacher, 1982) y El Faisanal, Gro. (Vargas et al., 1992). Además, 


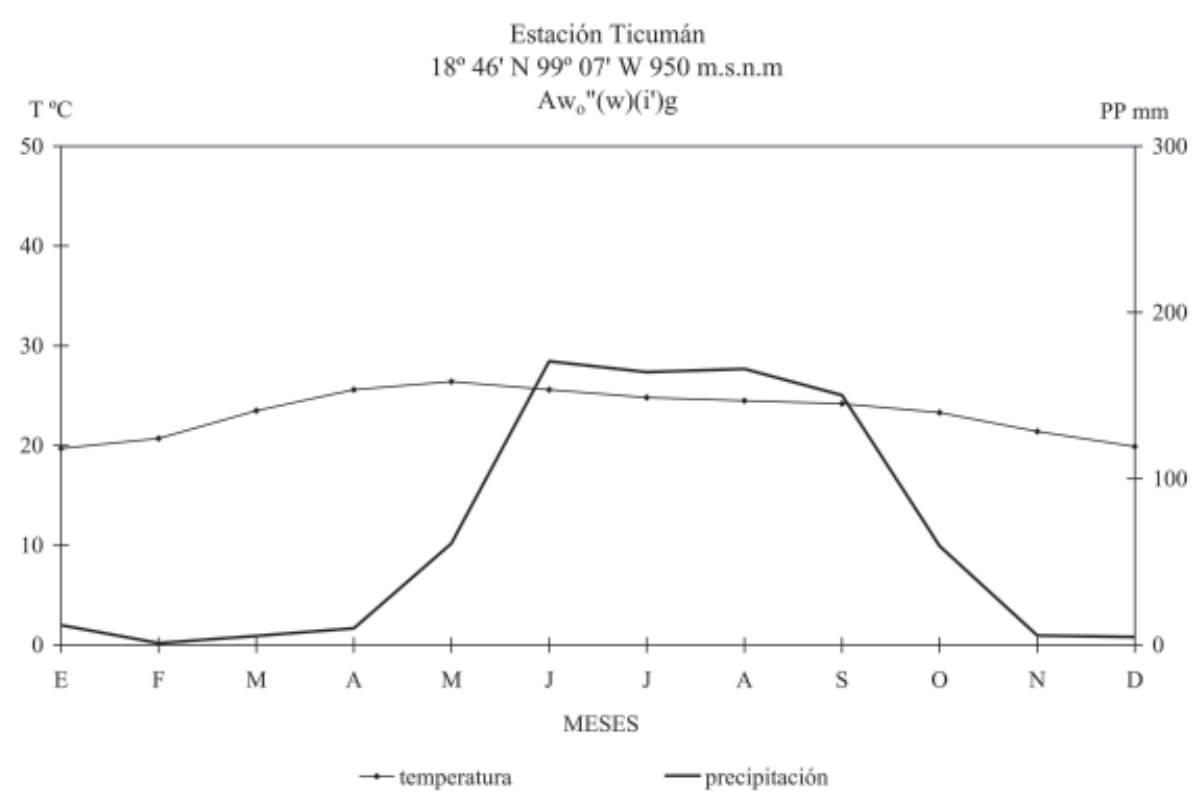

Figura 2. Marcha anual de la temperatura y distribución de la precipitación en la estación meteorológica de Ticumán, Morelos. Datos proporcionados por el Servicio Meteorológico Nacional.

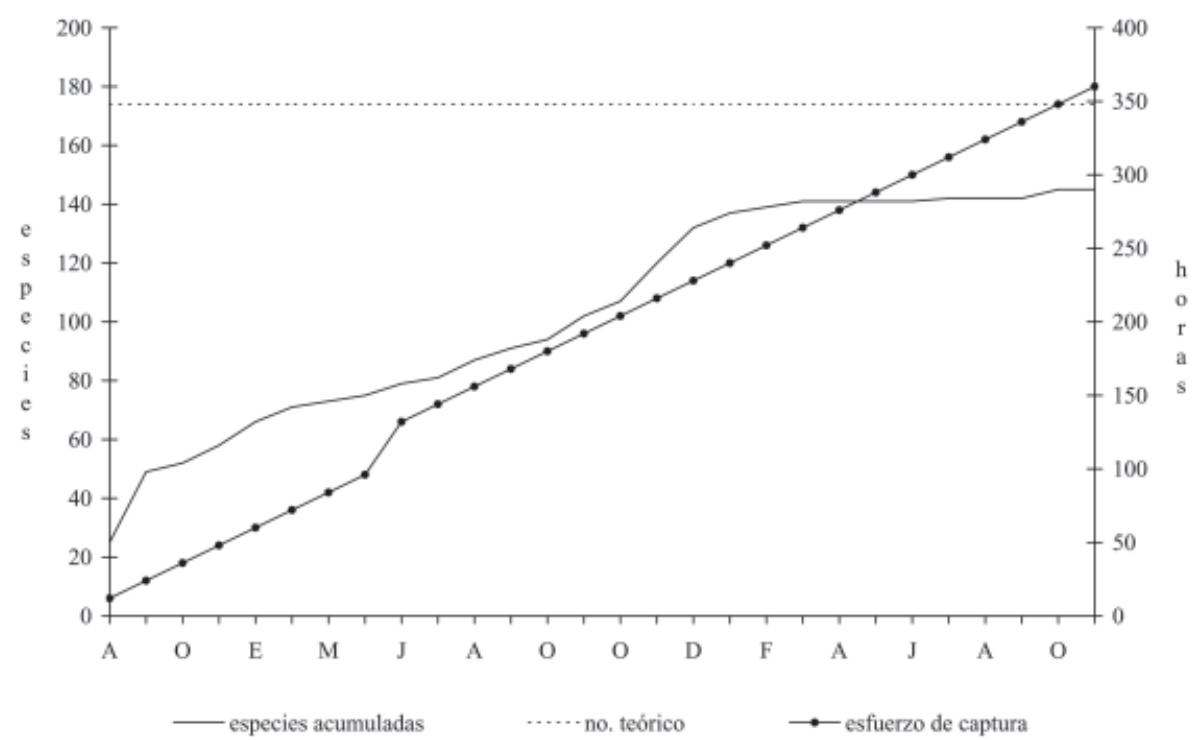

Figura 3. Curva de acumulación de especies. Esfuerzo de captura (horas/persona).

se contrastó con las listas de mariposas de otras áreas con otros tipos de vegetación, como Tepoztlán, Mor. (Maza, 1975) y Chichinautzin, Mor. (Valencia, 1999); El Chico, Hgo. (Bizuet et al., 2001) y Los Dínamos, Magdalena Contreras, D. F. (Luis y Llorente, 1990).

\section{Resultados}

Se obtuvo una lista integrada por 145 especies, incluidas en 85 géneros, 16 subfamilias y 4 familias de Papilionoidea (Apéndice $1)$; con base en la curva de acumulación de especies y la fórmula de Clench (1979), el número estimado de especies para el área de estudio es de 174 (Fig. 3).

La mayoría de las especies fueron Lycaenidae (54 especies agrupadas en 27 géneros) y Nymphalidae (53 spp. en 36 géneros), y en menor proporción Pieridae (24 spp. en 14 géneros) y Papilionidae (14 spp. de 8 géneros) (Apéndice 1); en cuanto a su abundancia, el $48 \%$ del total de ejemplares recolectados (3 708) fueron ninfálidos, el $30 \%$ piéridos, $13 \%$ licénidos y $9 \%$ papiliónidos (Cuadro 1).

En sitios equivalentes del estado de Morelos como Bastida, Rancho Viejo, Río del Pollo y Xochitepec (Maza et al., 1995b; Cuadro 2), la riqueza de Cañón de Lobos fue mayor; estas cañadas están ubicadas a una altitud similar (1050 a 1140 m snm), en áreas con los mismos tipos de vegetación y clima que Cañón de Lobos; excepto Bastida que se encuentra a mayor altitud (1290 m snm) y tiene un clima más fresco [A(C) $\mathrm{w}_{1}$ "(w)ig], y la localidad Río del Pollo que corresponde a un valle (Maza et al., 1995b).

En general, el patrón de variación mensual de la riqueza y el de la abundancia estándar fueron muy similares, es decir, en los meses en que hubo un aumento o disminución del número de especies correspondió el mismo cambio en el número de ejemplares. Durante los meses de junio 


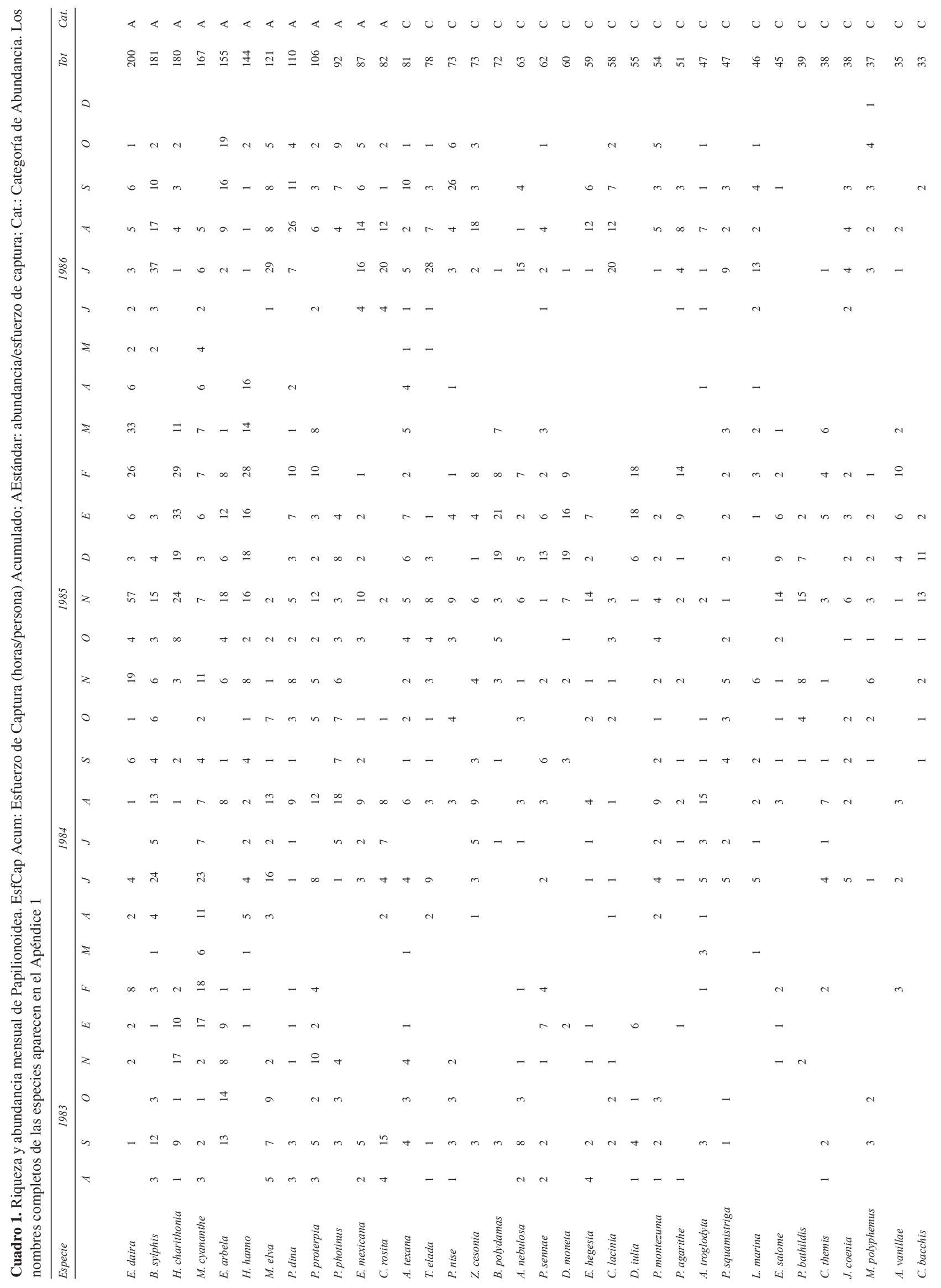




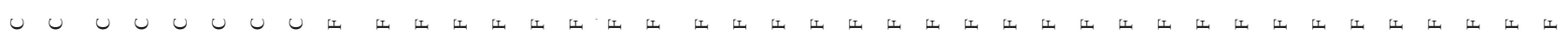

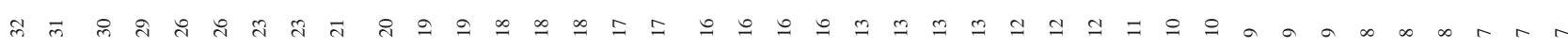

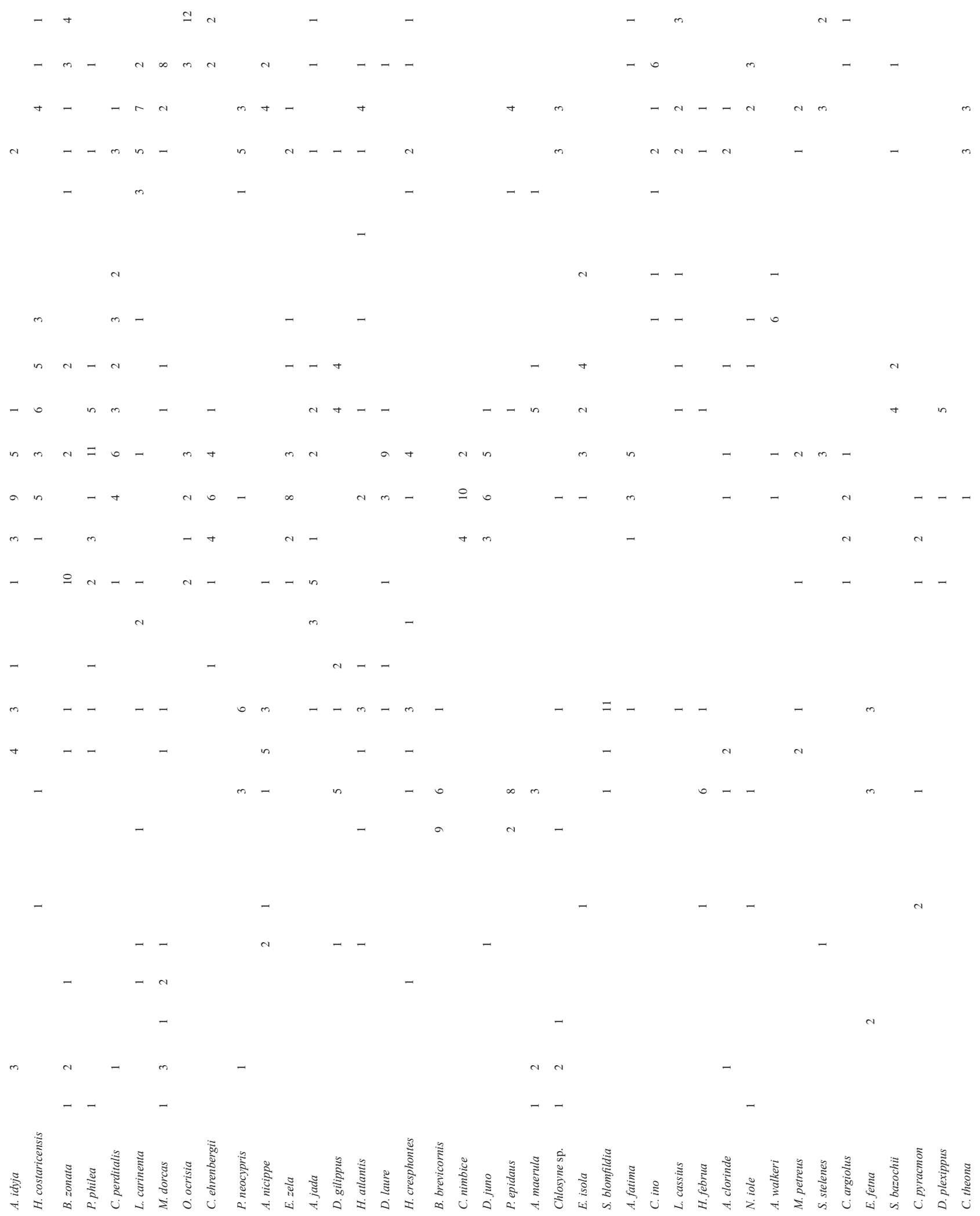




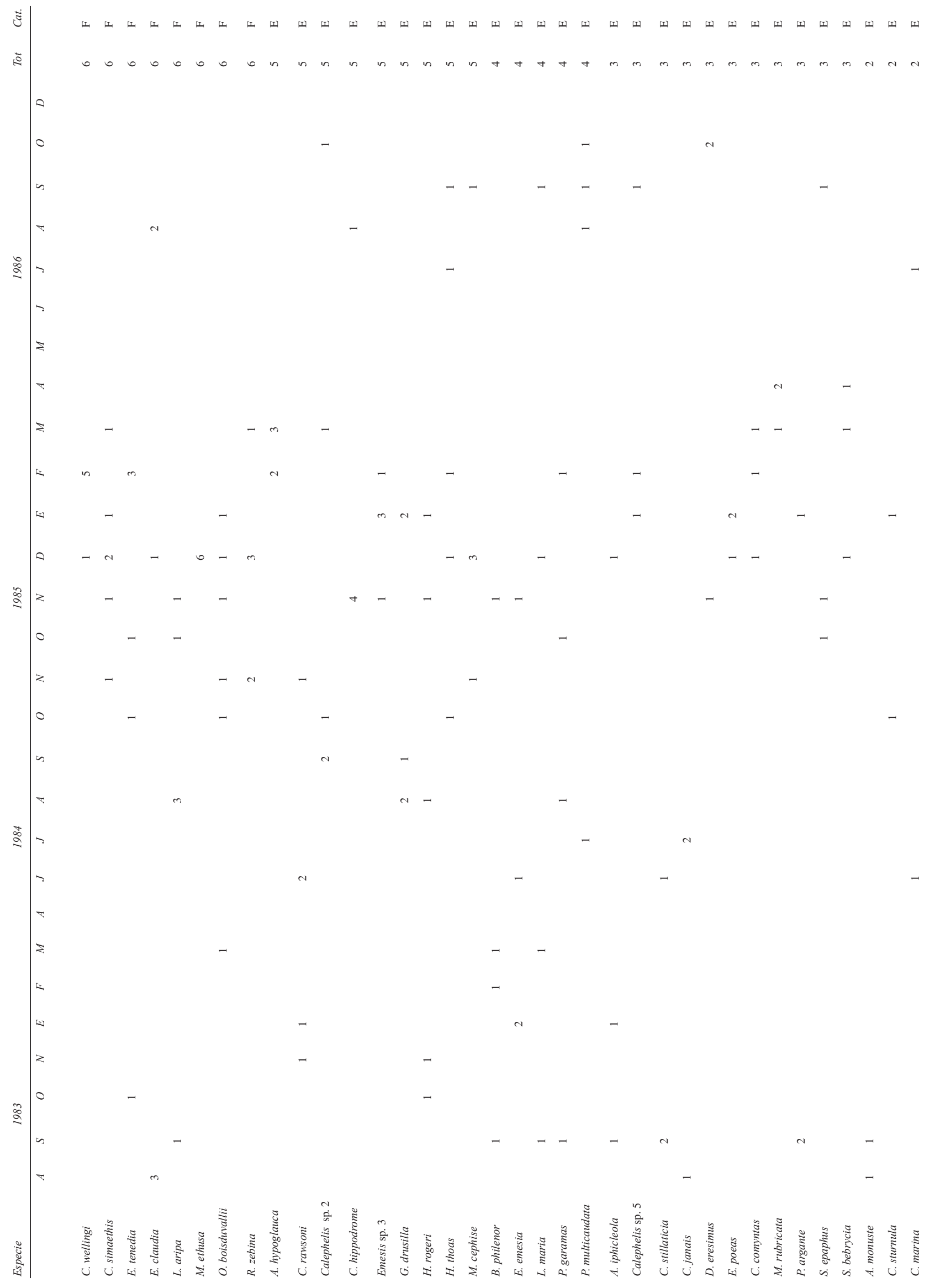




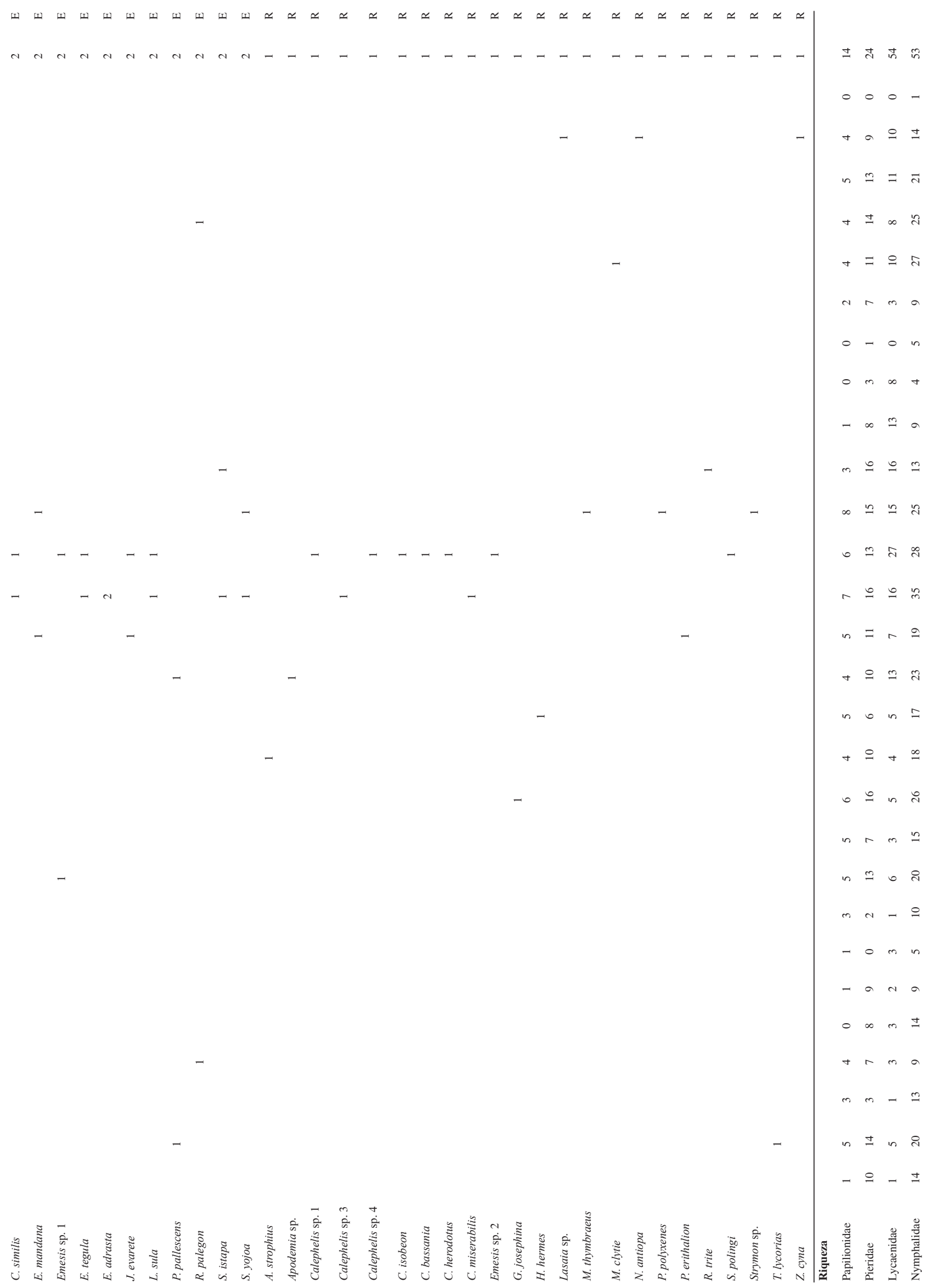


Cuadro 2. Riqueza de Cañón de Lobos y otras localidades del estado de Morelos, México

\begin{tabular}{|c|c|c|c|c|c|}
\hline Localidad & Papilionidae & Pieridae & Lycaenidae & Nymphalidae & Total \\
\hline $\begin{array}{l}\text { Bastida, } \\
\text { Yautepec }\end{array}$ & 15 & 27 & 32 & 66 & 140 \\
\hline $\begin{array}{l}\text { Cañón de } \\
\text { Lobos, } \\
\text { Yautepec }\end{array}$ & 14 & 24 & 54 & 53 & 145 \\
\hline $\begin{array}{l}\text { Rancho Viejo, } \\
\text { Tlaltizapán }\end{array}$ & 11 & 20 & 36 & 57 & 124 \\
\hline $\begin{array}{l}\text { Río del Pollo, } \\
\text { Xochitepec }\end{array}$ & 12 & 22 & 27 & 50 & 111 \\
\hline $\begin{array}{l}\text { Xochitepec, } \\
\text { Xochitepec }\end{array}$ & 12 & 23 & 36 & 59 & 130 \\
\hline $\begin{array}{l}\text { Acueducto, } \\
\text { Puente de } \\
\text { Ixtla }\end{array}$ & 15 & 24 & 48 & 75 & 162 \\
\hline
\end{tabular}

a noviembre se registró el número mayor de especies y de ejemplares, con valores inferiores entre diciembre y mayo, en especial durante este último mes; no obstante, los valores máximos de todo el estudio se presentaron entre octubre de 1985 y marzo de 1986 (Fig. 4). De acuerdo con la tabulación de resultados, durante el transcurso de los primeros meses de este trabajo se capturaron 1315 ejemplares de 102 especies, mientras que en la segunda mitad (octubre de 1985 a diciembre de 1986) se capturaron 2393 ejemplares de 132 especies (Cuadro 1).

Del total de especies, 12 fueron abundantes (A); entre ellas, Eurema daira sidonia, E. arbela boisduvaliana, Bolbonerura sylphis beatrix, Heliconius charithonia vazquezae y Myscelia c. cyananthe, con más de 150 ejemplares cada una; 31 especies fueron comunes (C), 40 frecuentes (F); 39 escasas (E) y 23 raras (R) (Cuadro 1; Fig. 5). En las categorías A y $\mathrm{C}$ se reunieron un tercio del total de las especies y más del $83 \%$ de los ejemplares de Papilionoidea, mientras que las otras categorías representaron en su conjunto el porcentaje restante, en el que la categoría $\mathrm{R}$ tuvo una contribución muy baja, con el $15 \%$ de las especies y el $0.6 \%$ del total de organismos. Las categorías A y $\mathrm{C}$ estuvieron constituidas principalmente por ninfálidos y piéridos, las categorías $\mathrm{F}$ y $\mathrm{E}$ por ninfálidos y licénidos, y la categoría $\mathrm{R}$ predominantemente por licénidos. Aunque en todas las categorías de abundancia están representados los papiliónidos, el número de especies y de ejemplares fue el más reducido de las 4 familias de Papilionoidea (Cuadro 1; Fig. 5).

La riqueza de Papilionoidea se incrementó desde la primavera hasta el otoño, estación en la cual se presentó el máximo número de especies; aun cuando en el invierno se redujo el número de especies, los valores más bajos ocurrieron durante la primavera (Fig. 6). Nymphalidae fue la familia más rica durante la mayor parte del año, excepto en el invierno cuando predominó Lycaenidae; así, durante la primavera, el otoño y el invierno la mayor proporción 


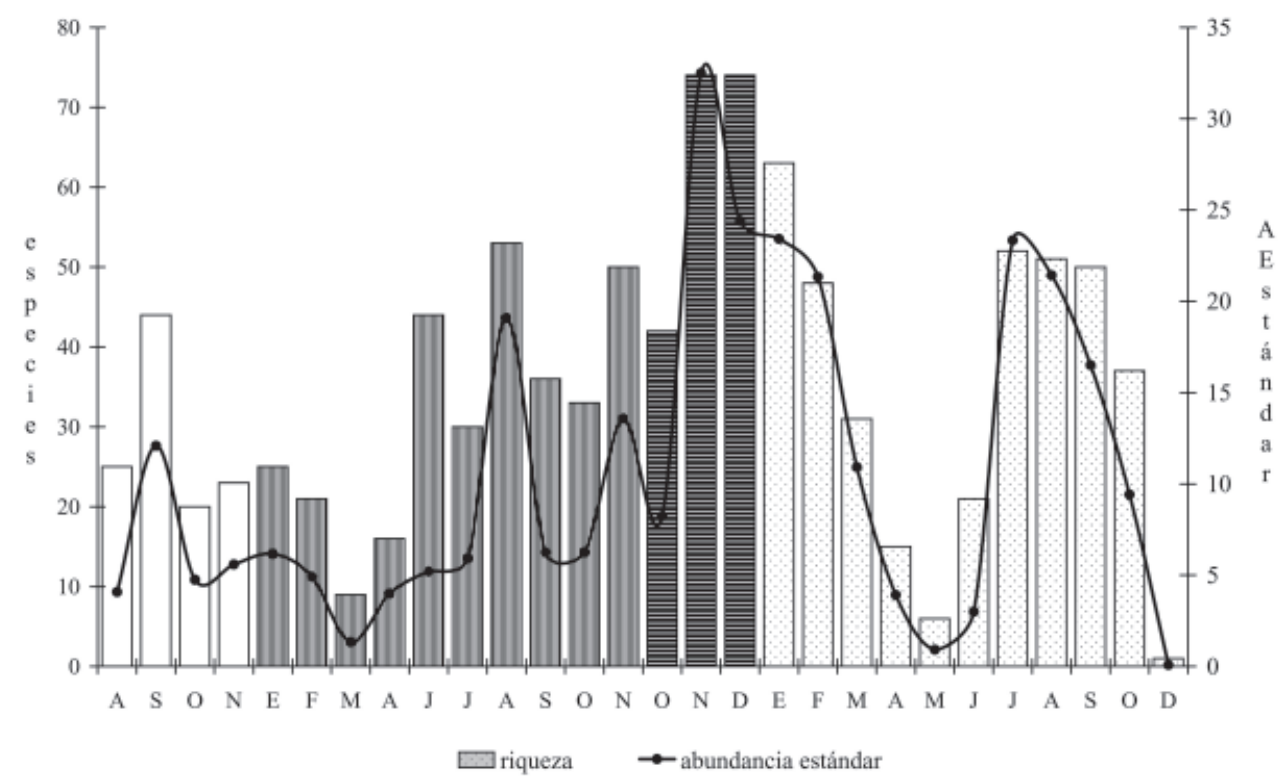

Figura 4. Distribución mensual de la riqueza y abundanciadePapilionoidea. AEstándar (número de ejemplares/ esfuerzo de captura). El achurado de las barras indica el año: 1983 (en blanco); 1984 (vertical); 1985 (horizontal); 1986 (punteado). de especies fueron ninfálidos y licénidos, mientras en el verano fueron piéridos y ninfálidos. Los valores máximos para cada familia ocurrieron en la segunda mitad del año; por ejemplo, Pieridae y Nymphalidae tuvieron más especies en el otoño, mientras Papilionidae y Lycaenidae durante el invierno. Por otro lado, la abundancia estándar (número de ejemplares/esfuerzo de captura) aumentó constantemente desde la primavera hasta el invierno, en el que los papiliónidos, ninfálidos y licénidos tuvieron las poblaciones mayores; durante el otoño los piéridos fueron más abundantes. Nymphalidae también fue la familia con mayor abundancia estándar durante las 4 estaciones (Fig. $6)$.

El 19.3\% de las especies (28) se presentó en todas las estaciones, aunque con mayor abundancia durante el verano, v. gr., los papiliónidos Battus p. polydamas y Parides montezuma, los piéridos E. daira sidonia y E. arbela boisduvaliana, los ninfálidos $B$. sylphis beatrix, $H$. charithonia vazquezae y M. c. cyananthe, y los licénidos Hemiargus hanno antibubastus y Leptotes marina (Fig. 7).

El resto de las especies estuvieron presentes en 3, en 2 o en 1 de las estaciones exclusivamente. Por ejemplo, el $30.4 \%$ de los papilionoideos (44 especies) se registró en 3 estaciones; la mayoría se encontraron durante todo el año, excepto en la primavera, que es el periodo más cálido y seco (Parides photinus, Eurema m. mexicana, Euptoieta hegesia meridiania, Arawacus jada y 27 especies más). Durante primavera, otoño e invierno (estaciones menos húmedas), volaron 7 especies, como Battus p. philenor, Echinargus isola y Apodemia walkeri; 5 especies se encontraron durante primavera, verano y otoño (periodo cálido y húmedo), como Microtia e. elva, Chlosyne rosita riobalsensis, Chlosyne l. lacinia y Caria i. ino. La única especie que se presentó en primavera, verano e invierno (meses húmedos y frescos) fue Protographium epidaus fenochionis (Cuadro 1).

Otro $26.9 \%$ (39 especies) estuvo presente en 2 estaciones. La mayoría durante otoño e invierno (mitad fresca del año), como Pantiades bathildis, Ocaria ocrisia, Cyclogramma bacchis, Chlosyne ehrenbergii, Danaus p. plexippus; 9 se capturaron únicamente en verano y otoño (la mitad húmeda), como Euptychia fetna, Pterourus $m$. multicaudata, Chlosyne theona y Leptophobia aripa elodia; los licénidos Apodemia h. hypoglauca, Strymon bebrycia y Cupido comyntas se capturaron durante primavera e invierno (mitad seca del año); Baronia b. brevicornis estuvo activa durante primavera y verano (mitad cálida del año), mientras Calephelis sp. 2, durante primavera y otoño. Por último, 3 especies se registraron durante el verano e invierno (meses más lluviosos y frescos), por ejemplo, Hamadryas februa ferentina y Euptoieta claudia daunius (Cuadro 1).

Finalmente, el $23.4 \%$ del total de especies (34, principalmente de licénidos) se registraron durante una estación. En invierno (estación seca y más fresca) se encontraron 14, en su mayoría licénidos, por ejemplo, Mimoides thymbraeus aconophos, Papilio polyxenes asterius, Rhabdodryas trite, Myscelia e. ethusa, Cyanophrys herodotus, Contrafacia bassania, Calycopis isobeon, Calephelis w. wellingi y Emesis poeas. Otras 14 se capturaron en otoño (estación húmeda y fresca), por 


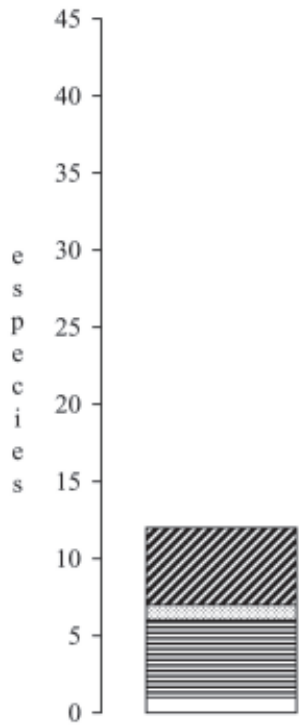

A

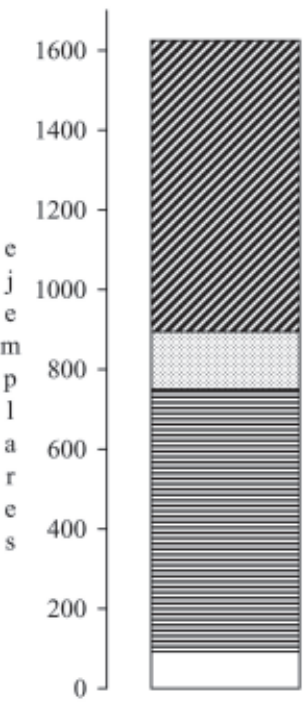

A

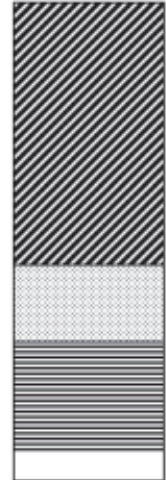

C

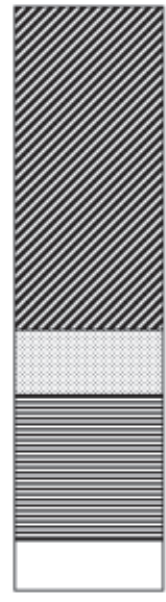

C

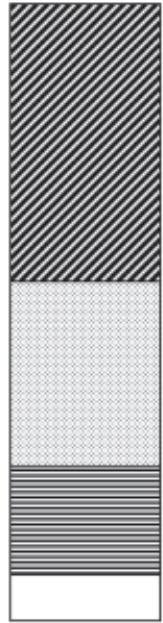

$\mathrm{F}$

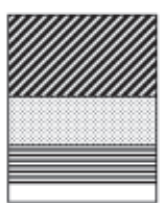

F

$\square$ Papilionidae 目Pieridae Q Lycaenidae $\boldsymbol{Z}$ Nymphalidae

Figura 5. Categorías de abundancia. A: especies abundantes; C: comunes; F: frecuentes; E: escasas; R: raras.

ejemplo, Parides erithalion trichopus, Danaus eresimus montezuma, Siproeta e. epaphus y Allosmaitia strophius. Algunas solamente se registraron en el verano (estación lluviosa), como Ganyra josephina josepha, Smyrna blomfildia datis y Ministrymon clytie; o como Megisto rubricata anabelae que únicamente fue capturada en primavera (estación cálida y seca) (Cuadro 1).

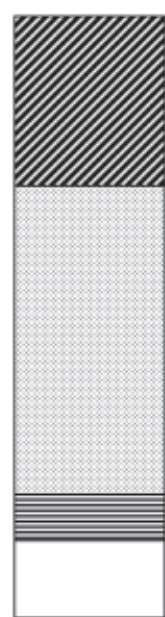

E

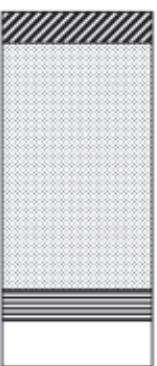

R

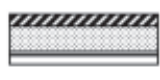

$\mathrm{R}$

Se reconocieron las plantas de alimentación larval de 118 especies de papilionoideos de Cañón de Lobos, con excepción de los que sólo se determinaron hasta nivel de género y la correspondiente a 13 especies para las que no se encontró información: $R$. trite, $C$. bassania, A. strophius, C. isobeon, Zizula cyna, C. w. wellingi, Caria stillaticia, Emesis mandana furor, E. poeas, $E$. tenedia, E. tegula, A. h. hypoglauca y $A$. walkeri.

De esta manera, se definieron 4 grupos distintos (Cuadro 3). El primero integrado por 14 especies de mariposas para las cuales se citó la especie de la planta huésped (E) en la zona de estudio: Heraclides cresphontes, Phoebis a. agarithe, $P$. $p$. philea, Pyrisitia dina westwoodi, $P$. nise nelphe, E. daira sidonia, C. herodotus, Strymon bazochii, L. marina, H. hanno antibubastus, E. isola, Danaus gilippus thersippus, D. p. plexippus y $C . \quad l$. lacinia.

Otro grupo estuvo conformado por 50 especies para las que se registró el género de la planta de alimentación larval en el área (G), como ocurrió con P. photinus, P. montezuma, B. p. polydamas, Pyrisitia proterpia, E. arbela boisduvaliana, P. bathildis, Dione moneta poeyi y $H$. charithonia vazquezae; adicionalmente, para $B$. b. brevicornis, Phoebis argante y Libytheana carinenta mexicana se citó 
RIQUEZA

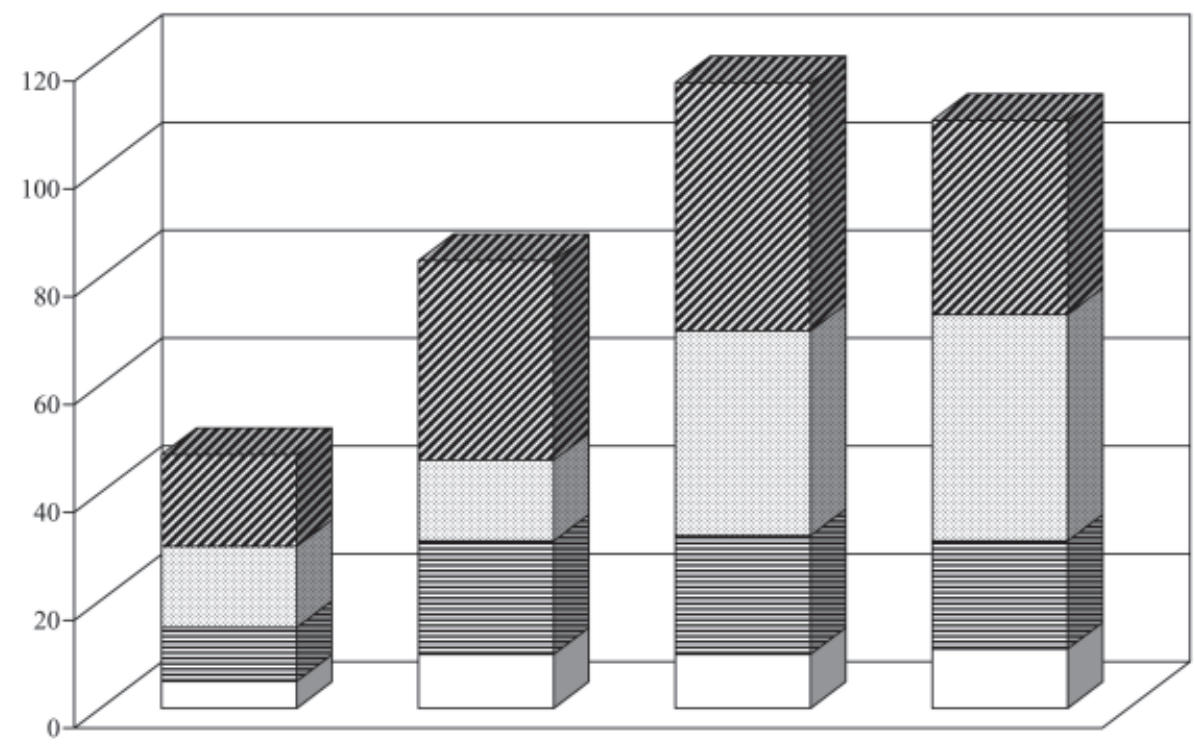

ABUNDANCIA

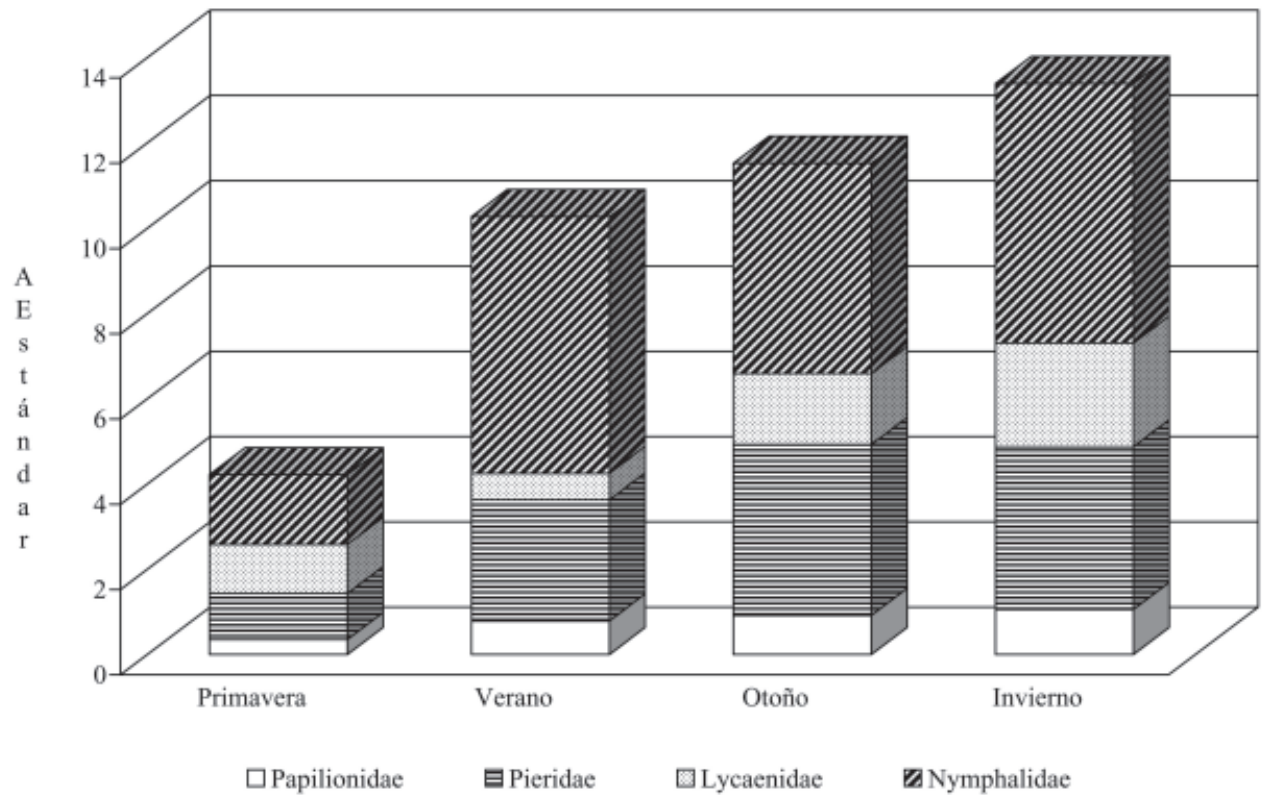

C. rosita riobalsensis y Anthanassa nebulosa alexon, por mencionar algunas de las más abundantes; en este grupo se incluyeron Siproeta stelenes biplagiata, S. blomfildia datis y Chlosyne j. janais, de las que también se citó la planta de alimentación en Morelos (F, EM), así como 13 especies, el género de cuya planta huésped se distribuye en esta entidad (F, GM), por ejemplo, Cyanophrys miserabilis, C. comyntas, Anthanassa t. texana, Nymphalis a. antiopa y S. e. epaphus.

El último conjunto incluyó13 papilionoideos de los cuales no hay registro de la especie, género o familia del huésped en Cañón de Lobos; no obstante, existe en el estado de Morelos, v. gr., para $P$. epidaus fenochionis, Hermeuptychia hermes y M. rubricata, se ha citado la especie de planta de alimentación (EM); el género (GM), en el caso de $M$. thymbraeus aconophos, Catasticta $n$. nimbice, Lasaia s. sula y Morpho p. polyphemus, y para Strymon istapa, Emeis z. zela y Chlosyne $h$. hippodrome se ha registrado la familia del huésped (FM).

Únicamente

8

especies fueron citadas en áreas de SBC: M. rubricata anabelae, Bolboneura sylphis beatrix, Calephelis rawsoni, Caria i. ino, así como Cyanophrys miserabilis, A. strophius, C. w. wellingi, y Junonia evarete nigrosuffusa; estas 3 últimas en Cañón de Lobos. 

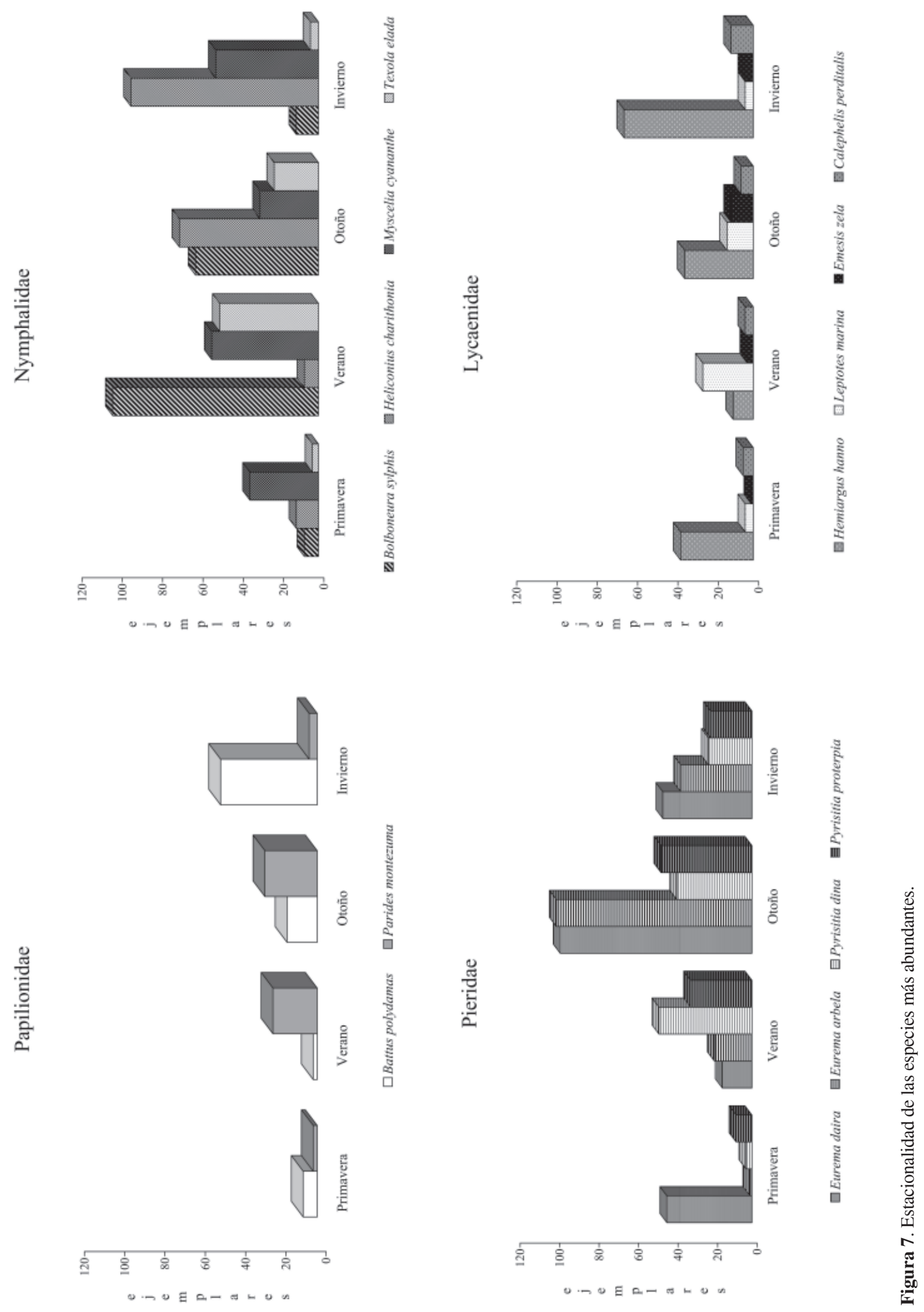
Cuadro 3. Huéspedes potenciales de los Papilionoidea de Cañón de Lobos. Planta huésped presente en la zona de estudio: E (especie), G (género), F (familia); planta huésped presente en el estado de Morelos: EM (especie), GM (género), FM (familia)

\begin{tabular}{|c|c|c|}
\hline Especie & Plantas de alimentación & Estatus \\
\hline \multicolumn{3}{|l|}{ PAPILIONIDAE } \\
\hline Baronia brevicornis & Acacia, Acacia cochliacantha & G, EM \\
\hline Papilio polyxenes asterius & Umbelliferae, Rutaceae, Daucus, Apium & F, GM \\
\hline Parides montezuma & Aristolochia & $\mathrm{G}$ \\
\hline Protographium epidaus & Annona reticulata & EM \\
\hline Mimoides thymbraeus & Annona & GM \\
\hline Heraclides cresphontes & Ptelea trifoliata & $\mathrm{E}$ \\
\hline Heraclides rogeri pharnaces & Citrus & $\mathrm{F}, \mathrm{GM}$ \\
\hline Heraclides thoas autocles & Citrus, Ptelea, Zanthoxylum, Piper & $\mathrm{G}$ \\
\hline Battus philenor & Aristolochia, Ipomoea & G \\
\hline Battus polydamas & Aristolochia & $\mathrm{G}$ \\
\hline Parides erithalion & Aristolochia & G \\
\hline Parides photinus & Aristolochia, Acacia, Dalea, Lysiloma, & $\mathrm{G}$ \\
\hline Pterourus garamas & Persea & GM \\
\hline Pterourus multicaudata & Ptelea & $\mathrm{G}$ \\
\hline \multicolumn{3}{|l|}{ PIERIDAE } \\
\hline Zerene cesonia & Indigofera & G \\
\hline Anteos clorinde & Cassia & G \\
\hline Anteos maerula & Cassia & G \\
\hline Phoebis agarithe & Pithecellobium dulce & $\mathrm{E}$ \\
\hline Phoebis argante & Cassia, Pithecellobium, Inga vera & G, EM \\
\hline Phoebis neocypris & Leguminosae & $\mathrm{F}$ \\
\hline Phoebis philea & Cassia leptocarpa & $\mathrm{E}$ \\
\hline Phoebis sennae marcellina & Cassia & G \\
\hline Abaeis nicippe & Cassia, Bidens & $\mathrm{G}$ \\
\hline Pyrisitia dina westwoodi & Alvaradoa amorphoides & $\mathrm{E}$ \\
\hline Pyrisita nise nelphe & Mimosa pudica & $\mathrm{E}$ \\
\hline Pyrisitia proterpia & Cassia & G \\
\hline Eurema arbela boisduvaliana & Cassia & G \\
\hline Eurema daira sidonia & Mimosa pudica & $\mathrm{E}$ \\
\hline Eurema mexicana & Cassia, Diphysa & $\mathrm{G}$ \\
\hline
\end{tabular}




\begin{tabular}{|c|c|c|}
\hline Especie & Plantas de alimentación & Estatus \\
\hline Eurema salome & Diphysa & G \\
\hline Nathalis iole & Bidens & $\mathrm{G}$ \\
\hline Hesperocharis costaricensis & Cruciferae & $\mathrm{F}$ \\
\hline Catasticta nimbice nimbice & Phoradendron & GM \\
\hline Glutophrissa drusilla & Cassia & G \\
\hline Leptophobia aripa & Brassica & G \\
\hline Ascia monuste & Brassica & G \\
\hline Ganyra josephina & Capparis & GM \\
\hline \multicolumn{3}{|l|}{ LYCAENIDAE } \\
\hline Rekoa palegon & Caesalpinia & G \\
\hline Rekoa zebina & Caesalpinia & G \\
\hline Arawacus jada & Solanum & $\mathrm{G}$ \\
\hline Satyrium polingi & Quercus & GM \\
\hline Ocaria ocrisia & Caesalpinia & G \\
\hline Chlorostrymon simaethis & Eupatorium & $\mathrm{F}, \mathrm{GM}$ \\
\hline Cyanophrys herodotus & Lantana camara & $\mathrm{E}$ \\
\hline Cyanophrys miserabilis & Leguminosae, Parkinsonia & $\mathrm{F}, \mathrm{GM}$ \\
\hline Strymon bebrycia & Sapindaceae & $\mathrm{F}$ \\
\hline Strymon yojoa & Leguminosae, Desmodium & $\mathrm{F}, \mathrm{GM}$ \\
\hline Strymon bazochii & Lippia graveolens & $\mathrm{E}$ \\
\hline Strymon istapa & Malvaceae & FM \\
\hline Ministrymon clytie & Leguminosae, Prosopis & $\mathrm{F}, \mathrm{GM}$ \\
\hline Panthiades bathildis & Caesalpinia & G \\
\hline Leptotes cassius cassidula & Pithecellobium, Indigofera & $\mathrm{G}$ \\
\hline Leptotes marina & Eysenhardtia polystachya & $\mathrm{E}$ \\
\hline Cupido comyntas & Leguminosae, Desmodium, Medicago & $\mathrm{F}, \mathrm{GM}$ \\
\hline Celastrina argiolus gozora & Erythrina, Verbesina & $\mathrm{G}$ \\
\hline Hemiargus hanno antibubastus & Acacia angustissima & $\mathrm{E}$ \\
\hline Echinargus isola & Acacia angustissima & $\mathrm{E}$ \\
\hline Calephelis perditalis perditalis & Compositae, Eupatorium & $\mathrm{F}, \mathrm{GM}$ \\
\hline Calephelis rawsoni & Eupatorium & F, GM \\
\hline Caria ino ino & Celtis, Tillandsia & $\mathrm{G}$ \\
\hline Baeotis zonata zonata & $\begin{array}{l}\text { Bromeliaceae, Boraginaceae, Poligonaceae, Labiatae, Solanaceae, } \\
\text { Piperaceae, Convolvulaceae, Oxaliadaceae }\end{array}$ & $\mathrm{F}$ \\
\hline
\end{tabular}




\begin{tabular}{lll} 
Especie & Plantas de alimentación & Estath \\
\hline Lasaia sula sula & Albizzia \\
Lasaia maria & Bromeliaceae, Boraginaceae, Poligonaceae, Labiatae, Solanaceae, \\
Melanis cephise acroleuca & Piperaceae, Convolvulaceae, Oxaliadaceae \\
Calydna sturnula & Bromeliaceae, Boraginaceae, Poligonaceae, Labiatae, Solanaceae, \\
Emesis zela cleis & Piperaceae, Convolvulaceae, Oxaliadaceae \\
Emesis emesia & Bromeliaceae, Boraginaceae, Poligonaceae, Labiatae, Solanaceae, \\
Thisbe lycorias & Piperaceae, Convolvulaceae, Oxaliadaceae \\
& Fagaceae \\
& Caesalpinia \\
& Caesalpinia
\end{tabular}

\section{NYMPHALIDAE}

Libytheana carinenta

Danaus eresimus

Danaus gilippus

Danaus plexippus

Morpho polyphemus

Opsiphanes boisduvalii

Cissia similis

Cissia themis

Cyllopsis pyracmon pyracmon

Euptychia fetna

Hermeuptychia hermes

Megisto rubricata anabelae

Pindis squamistriga

Anaea troglodyta

Marpesia petreus

Mestra dorcas

Myscelia cyananthe

Myscelia ethusa

Hamadryas atlantis

Hamadryas februa

Bolboneura sylphis beatrix

Epiphile adrasta adrasta

Cyclogramma bacchis

Adelpha iphicleola

Asterocampa idyja argus
Celtis, Symphoricarpus occidentalis

G, EM

Sarcostemma clausum

Asclepias curassavica

Asclepias curassavica

Sapindaceae, Inga

F, GM

Asclepiadaceae y Apocynaceae

Ficus, Spondias, Guazuma

Asclepiadaceae, Apocynaceae, Gramineae $\quad$ F

Asclepiadaceae, Apocynaceae $\quad$ F

Asclepiadaceae, Apocynaceae $\quad$ F

Cynodon dactylon $\quad$ EM

Cynodon dactylon $\quad$ EM

Asclepiadaceae, Apocynaceae, Gramineae, Cyperaceae $\quad$ F

Croton, Acalypha G

Ficus $\quad$ G

Euforbiaceae $\quad F$

Euforbiaceae $\quad F$

Euforbiaceae $\quad$ F

Euforbiaceae $\quad F$

Euforbiaceae $\quad$ F

Asclepiadaceae, Apocynaceae $\quad$ F

Serjania $\quad$ G

Sapindaceae $\quad$ F

Rubiaceae, Moreaceae, Urticaceae, Verbenaceae, Bombacaceae, $\quad$ F Piperaceae, Tiliaceae, Ericaceae Celtis
EM

E

E

F

G

M

F

F

M

G

G

F

F

F

M

M

F

G

G




\begin{tabular}{|c|c|c|}
\hline Especie & Plantas de alimentación & Estatus \\
\hline Doxocopa laure laure & Celtis & $\mathrm{G}$ \\
\hline Smyrna blomfildia & Urrera baccifera, U. caracasana, U. mexicanum & $\mathrm{F}, \mathrm{EM}$ \\
\hline Nymphalis antiopa antiopa & Ulmaceae, Moraceae, Tiliaceae, Salix, Alnus & F, GM \\
\hline Anartia fatima fatima & Dicliptera & $\mathrm{G}$ \\
\hline Junonia coenia & Lippia & $\mathrm{G}$ \\
\hline Junonia evarete nigrosuffusa & Lippia & $\mathrm{G}$ \\
\hline Siproeta epaphus epaphus & Acanthaceae, Ruellia, Blechum & $\mathrm{F}, \mathrm{GM}$ \\
\hline Siproeta stelenes & Acanthaceae, Blechum brownei & $\mathrm{F}, \mathrm{EM}$ \\
\hline Chlosyne ehrenbergii & Buddleia & GM \\
\hline Chlosyne hippodrome & Asteraceae & FM \\
\hline Chlosyne janais & Anisacanthus wrightii & F, EM \\
\hline Chlosyne lacinia & Viguiera dentata & $\mathrm{E}$ \\
\hline Chlosyne marina & Asclepiadaceae, Apocynaceae & $\mathrm{F}$ \\
\hline Chlosyne rosita riobalsensis & Asclepiadaceae, Apocynaceae & $\mathrm{F}$ \\
\hline Chlosyne theona & Castilleja & GM \\
\hline Microtia elva & Asclepiadaceae, Apocynaceae & $\mathrm{F}$ \\
\hline Texola elada & Baccharis & G \\
\hline Anthanassa nebulosa alexon & Asclepiadaceae, Apocynaceae & $\mathrm{F}$ \\
\hline Anthanassa texana & Acanthaceae, Dicliptera, Jacobina, Ruellia & F, GM \\
\hline Phyciodes pallescens & Asclepiadaceae, Apocynaceae & $\mathrm{F}$ \\
\hline Euptoieta claudia & Passiflora, Metastelma & G \\
\hline Euptoieta hegesia & Passiflora & $\mathrm{G}$ \\
\hline Agraulis vanillae & Passiflora & G \\
\hline Dione juno & Passiflora & $\mathrm{G}$ \\
\hline Dione moneta & Passiflora & $\mathrm{G}$ \\
\hline Dryas iulia & Passiflora & $\mathrm{G}$ \\
\hline Heliconius charithonia & Passiflora & $\mathrm{G}$ \\
\hline
\end{tabular}

\section{Discusión}

Lista de especies. La revisión de la literatura reveló que 13 especies ya habían sido registradas con anterioridad en la zona de estudio: B. b. brevicornis, B. p. philenor, P. montezuma, P. photinus, Heraclides rogeri pharnaces, C. miserabilis, P. bathildis, Calephelis yautepequensis, A. h. hypoglauca, Paramacera x. xicaque, M. c. cyananthe, Hamadryas atlantis lelaps y $H$. glauconome grisea, (Nicolay, 1976; Maza y Turrent, 1977; Miller, 1978; Clench, 1981; Jenkins, 1983; Beutelspacher, 1984; Jenkins, 1984; Ferris, 1985); con excepción de C. yautepequensis, P. $x$. xicaque y $H$. glauconome grisea todas fueron capturadas en este trabajo, de tal forma que la lista completa de los 
Papilionoidea de Cañón de Lobos es de 148 especies.

Del total, $14 \%$ de los taxones en el nivel de especie (21) son endémicos de México: B. b. brevicornis, P. epidaus fenochionis, M. thymbraeus aconophos, P. erithalion trichopus, Pterourus g. garamas, C. n. nimbice, E. fetna, P. x. xicaque, A. nebulosa alexon, C. ehrenbergii, Chlosyne m. marina, C. rosita riobalsensis, Phyciodes pallescens, Texola e. elada, H. atlantis lelaps, H. glauconome grisea, B. sylphis beatrix, C. yautepequensis, C. stillaticia, Melanis cephise acroleuca y E. poeas (Luis et al., 2003b); además, B. b. brevicornis, B. sylphis beatrix, así como M. e. elva, pueden considerarse especies muy antiguas, éstas son aceptadas por Maza (1987) como "reliquias". De acuerdo con este autor, la SBC es una vegetación caracterizada por la presencia de especies neotropicales antiguas y por su gran proporción de endemismo.

Esfuerzo de captura. La riqueza obtenida puede representar el $83.8 \%$ teórico de los Papilionoidea que habitan Cañón de Lobos, lo cual coincide con el análisis del esfuerzo de captura; el número de especies nuevas aumentó constantemente en la mayor parte del estudio, pero fue menor en los últimos meses. A pesar que el número acumulado de horas por persona fue cada vez mayor (Cuadro 1), aún falta registrar algunos papilionoideos. Con la adición a la lista de los 3 registros obtenidos de la literatura, esta cifra puede reducirse a 26 especies; sólo en casos extraordinarios se puede enumerar la totalidad de las especies presentes en un sitio (Cam et al., 2002).

Riqueza comparativa de Papilionoidea. Cañón de Lobos es una de las cañadas de Morelos con mayor riqueza. Las localidades de Acueducto, Bastida, Rancho Viejo, Río del Pollo y Xochitepec tienen características climáticovegetacionales equivalentes a las de Cañón de Lobos, no obstante, tal vez las condiciones propias de cada cañada influyan en la riqueza y composición de la ropalocerofauna. De acuerdo con Maza et al. (1995b), la combinación de algunos factores topográficos como el relieve accidentado, la profundidad, el 'respaldo altitudinal' (distancia entre la sima y la cumbre más alta del área adyacente al sitio estudiado), así como la orientación (exposición hacia un punto cardinal), incrementan la variación microclimática y de hábitats representados. De todos estos factores, parece ser que principalmente el respaldo "altitudinal", la orientación y en menor medida el relieve, condicionan una riqueza mayor de la ropalocerofauna en una cañada. En este sentido, Maza et al. (1995b) encontraron que el número mayor de especies de papilionoideos se presentó en Acueducto, que es la cañada con mayor respaldo altitudinal $(1400 \mathrm{~m})$, con exposición hacia el norte y relieve escabroso. Es probable que la riqueza alta encontrada en Cañón de Lobos también se deba a estos factores pues tiene la misma orientación y el mismo tipo de relieve; aunque no se cuenta con el dato del respaldo altitudinal, el área de estudio forma parte y es adyacente a la sierra de Yautepec (Fig. 1), macizo montañoso con elevaciones de más de 1700 m, lo cual permite suponer que en Cañón de Lobos existe un amplio respaldo altitudinal como el que se presenta en la cañada Acueducto.

La riqueza encontrada en este trabajo representa el 41 $\%$ de la fauna de Papilionoidea reconocida para Morelos (Luna-Reyes, 2007a), lo cual indica que Cañón de Lobos es una de las áreas más ricas de la entidad al compararla con otros estudios (Maza, 1975; Maza et al., 1995a, 1995b).

La proporción de especies por familia en Cañón de Lobos coincide con la encontrada en la mayor parte del país, e incluso con toda la región neotropical (Luis et al., 2003b), donde Nymphalidae y Lycaenidae siempre representan las familias más diversas, después Pieridae y al final Papilionidae. El número de especies de papiliónidos y piéridos fue similar entre Cañón de Lobos y Bastida, Rancho Viejo, Río del Pollo, Xochitepec y Acueducto, en contraste, tuvo uno de los valores más bajos de ninfálidos, así como la mayor riqueza de licénidos (54 especies), diferencia que constituye alrededor del $20 \%$ más con respecto a Acueducto, o el doble de especies de esta familia con respecto a Río del Pollo (Cuadro 2).

Los ninfálidos y licénidos suman 2 tercios o más de la riqueza total de papilionoideos en el mundo y en el Neotrópico, como se ha encontrado también a distintas escalas en otros trabajos desarrollados en diferentes áreas con condiciones altitudinales y climático-vegetacionales similares, tales como Chamela (Beutelspacher, 1981), Chorros del Varal (Arteaga, 1991), Pedernales (Balcázar, 1993) y la sierra de Huautla (Luna-Reyes et al., 2008), o con características distintas, como Los Dínamos (Luis y Llorente, 1990), Chichinautzin (Valencia, 1999), El Chico (Bizuet et al., 2001), así como los realizados en regiones con gradientes altitudinales más amplios, como la Huasteca potosina (Maza y White, 1990), la sierra de Juárez (Luis et al., 1991), sierra de Atoyac (Vargas et al., 1992), Omiltemi (Luis y Llorente, 1993), sierra de Manantlán (Vargas et al., 1999) y sierra Nevada (Luna-Reyes y Llorente, 2004). En Cañón de Lobos y Omiltemi el porcentaje de licénidos es tan alto como el de ninfálidos, mientras que en otros sitios con la misma vegetación pero menos cálidos como Bastida, o más secos como Río del Pollo, la proporción de licénidos es notablemente menor. Chamela, ubicada en el clima más seco (BS), representa el caso extremo en el que el porcentaje de licénidos apenas es un tercio de la riqueza de Nymphalidae. Comúnmente la mayor diversidad de Lycaenidae está asociada a condiciones altas de temperatura y humedad, por lo que es probable que la humedad sea el factor principal que explique la mayor proporción de licénidos en Cañón de Lobos. 
Distribución mensual de los papilionoideos. La riqueza y la abundancia fueron menores durante la etapa de construcción de la carretera Cuernavaca-Cuautla, en comparación con los valores de los meses posteriores. Con este camino se eliminó una parte importante de la vegetación en la que frecuentemente se observaron numerosas mariposas; la superficie afectada fue una franja de $12 \mathrm{~m}$ a lo largo de $4 \mathrm{~km}$ en una de las partes más bajas del cañón. Al parecer, la deforestación de las 4.8 has de selva tuvo un impacto negativo en la composición de la ropalocerofauna y sus poblaciones pues, en el último periodo, los papilionoideos presentaron una distribución más definida de la riqueza y abundancia, probablemente en respuesta a la recuperación de la dinámica de la comunidad de mariposas, 2 años después que el terreno fue desprovisto de parte de la vegetación original (Cuadro 1). Las mariposas están estrechamente relacionadas con las plantas, y su presencia en un sitio depende de la flora y de la estructura de la vegetación (Shapiro, 1974); las alteraciones en esta última tienen un efecto importante en la ropalocerofauna, principalmente sobre las especies residentes pues se modifica la disponibilidad de plantas de alimentación larval y del adulto (Moore, 1975).

Los valores de riqueza y abundancia que se obtuvieron en el semestre de octubre de 1985 a marzo de 1986 son los más altos para todo el estudio; resultan extraordinarios en comparación con otras áreas de la cuenca del Balsas cuyas características climático-vegetacionales son equivalentes. Estos meses (excepto octubre) corresponden a la temporada seca, cuando el número de especies y de ejemplares se reduce sustancialmente, como en el caso de Chorros del Varal (Arteaga, 1991) y Pedernales (Balcázar, 1993) en Michoacán, o en la sierra de Huautla en Morelos (Luna-Reyes et al., 2008). Con probabilidad, esta riqueza y abundancia atípicas pueden estar relacionadas con el fenómeno periódico El Niño. Durante este evento, casi cada 4 años, en México se modifican los regímenes de lluvias, el invierno es más frío y húmedo, mientras el verano es más seco y cálido; la disminución de la precipitación tiene repercusiones principalmente en la región central del país, pues en el verano la sequía es más severa, alterando la vegetación de los hábitats tropicales áridos como la SBC (Mosiño y Morales, 1988). Uno de los eventos más intensos y largos en los últimos 50 años ocurrió entre noviembre de 1982 y agosto de 1984, y otro más débil y con menores consecuencias se presentó desde julio de 1986 al primer trimestre de 1988 (ENSO, 2006; Magaña et al., 2006); sin embargo, su periodicidad no siempre es regular, y su duración e influencia puede ser mayor.

Precisamente entre estos 2 eventos de El Niño se encontraron los valores más altos de riqueza y abundancia de papilionoideos en Cañón de Lobos (Fig. 4); además del restablecimiento natural de la vegetación que se mencionó anteriormente, es posible que las condiciones más húmedas durante el verano hayan favorecido el incremento de la diversidad y la biomasa de la comunidad vegetal, lo cual puede propiciar el establecimiento de más especies y poblaciones mayores de Papilionoidea (Rhoades, 1983).

La riqueza y la abundancia más bajas se presentaron durante los meses de marzo a mayo (Fig. 4), que es la mitad del año en la que se presenta la mayor oscilación de la temperatura $\left(5.9^{\circ} \mathrm{C}\right.$ de diferencia entre el mes más frío y el más cálido), así como el mayor número de días despejados, y por consiguiente la mayor evaporación. Bajo estas condiciones, la mayor parte de la vegetación está seca, sobre todo algunas plantas herbáceas que, en floración, proporcionan alimento a los imagos; durante la sequía también disminuyen las reservas hídricas de las especies arbóreas y arbustivas modificando su crecimiento, la producción de néctar, el contenido nutricional o incluso la textura y turgencia de las hojas que constituyen recursos alimenticios de las larvas y adultos de la mayoría de los papilionoideos. Aunque los árboles y arbustos están presentes en el hábitat, por su fenología caducifolia, muchos de ellos no pueden utilizarse por las mariposas durante este periodo, afectando de esta forma la composición de la comunidad de mariposas y sus poblaciones en estos meses.

Por el contrario, en la mitad húmeda del año (mayo a octubre) la temperatura es más estable $\left(3.1^{\circ} \mathrm{C}\right.$ entre el mes más frío y el más cálido), pero las condiciones de humedad son notablemente superiores y contrastantes con respecto a la época seca, en la que la precipitación total es de $39.2 \mathrm{~mm}$, mientras que en la temporada de lluvias es de $772 \mathrm{~mm}$. Aunque las primeras lluvias tienen lugar hacia finales de mayo, la precipitación mayor se presenta de junio a septiembre, y como consecuencia existe mayor nubosidad que reduce la evaporación; durante esta época, la vegetación es más variada y exuberante, y proporciona una mayor cantidad de recursos que son utilizados por las mariposas para su alimentación, oviposición, protección, percheo y patrullaje, características que favorecen la presencia de más especies de papilionoideos con mayores poblaciones. Sin embargo, existe un desfasamiento entre los meses de mayor precipitación (principios de junio a octubre) y el periodo de mayor riqueza y abundancia de la ropalocerofauna (finales de junio a noviembre). Las mariposas de Cañón de Lobos tienen mayor actividad cuando las condiciones meteorológicas son menos adversas para ellas (lluvia y nubosidad intensas) y la fenología de la vegetación está en condiciones óptimas, como también se ha registrado de otras localidades (Luis y Llorente, 1990).

A diferencia de las zonas templadas, en las que el fotoperiodo y la temperatura influyen en la dinámica 
de la comunidad de Papilionoidea, en las áreas tropicales la longitud del día y la temperatura no varían significativamente, por lo que la mayor actividad de las mariposas depende en especial de la presencia de las lluvias (Owen, 1971), mostrando una marcada estacionalidad con máximos de riqueza y abundancia al principio, durante o después de la época de lluvias, y valores mínimos en la temporada seca (Owen, 1971; Austin, 1978; Rodríguez, 1982; Ehrlich, 1984; Morón y Terrón, 1984; Wolda, 1988; Balcázar, 1993), como ocurre en Cañón de Lobos.

Algunos patrones estacionales relacionados con el clima requieren conocer el ciclo de vida completo de cada una de las especies involucradas (Wolda, 1988). Por ejemplo, en este estudio, la mayor concentración de especies y ejemplares se presentó en la temporada húmeda, pero algunos papilionoideos únicamente fueron comunes en un periodo bien definido; por ejemplo, C. w. wellingi, E. poeas, $C$. isobeon y 13 especies más de licénidos que fueron registrados en los meses secos; este patrón temporal de licénidos también se ha encontrado en otras áreas tropicales (Owen, 1971). Otras especies, como E. daira sidonia, P. nise nelphe, M. c cyananthe, B. sylphis beatrix y $A$. t. texana tuvieron amplia distribución temporal, pues se presentaron en 22 de los 28 meses estudiados y pueden considerarse multivoltinas.

Abundancia por especie. En general, los licénidos se agrupan entre las especies escasas o raras debido a su tamaño poblacional reducido, mientras que los ninfálidos y piéridos ocupan las categorías de mayor abundancia. A menudo se encuentran pocas especies abundantes y muchas raras que contienen una proporción pequeña de la población total (Clench, 1979; Smith, 1980); sin embargo, en Cañón de Lobos la proporción de especies raras fue relativamente baja. Estos resultados coinciden con los obtenidos en Los Dínamos (Luis y Llorente, 1990) y en la sierra de Huautla (Luna-Reyes et al., 2008), puesto que la mayor concentración de especies se presentó en las categorías de abundancia que comprendieron de 2 a 5 y de 6 a 21 registros (categorías $\mathrm{E}$ y $\mathrm{F}$ de este trabajo). A diferencia de las otras localidades, ninguna especie de Cañón de Lobos correspondió a la categoría MA (muy abundante; más de 332 registros), ya que la especie más abundante fue E. daira sidonia con 200 ejemplares.

Estacionalidad. Los papilionoideos multivoltinos a menudo se registraron en otoño e invierno con números de población mayores, cuando las condiciones climáticas en Cañón de Lobos son menos extremas (al final de la época de lluvias); aquellas especies multivoltinas que estuvieron presentes en el verano tuvieron durante éste una abundancia comparativamente menor.

El 10\% de la ropalocerofauna se registró en todas las estaciones, pero con máximos poblacionales durante el verano y el otoño (mitad húmeda), como el caso de $P$. montezuma, $P$. dina westwoodi, $P$. proterpia, $B$. sylphys beatrix, H. charithonia vazquezae, T. e. elada y L. marina (Fig. 7). Otro grupo reducido de especies prefiere condiciones más secas pues fueron más activas durante la primavera y el invierno, por ejemplo, H. hanno antibubastus y E. z. zela (Fig. 7), lo cual sugiere que la estación húmeda no siempre es la más favorable para todas las especies de mariposas, como generalmente se afirma (Owen, 1971).

Los papilionoideos de Cañón de Lobos también mostraron el comportamiento de distribución temporal que se registra en áreas tropicales (Arteaga, 1991; Luis et al., 1991; Vargas et al., 1992, 1999; Balcázar, 1993), en la que los imagos son más activos durante las estaciones de verano y otoño, es decir, durante la mitad húmeda del año, cuando la disponibilidad de recursos es mayor (Courtney, 1986). En cambio, en las zonas templadas el fotoperiodo y la temperatura propician los valores máximos en la primavera (Shapiro, 1974, 1975; Scott y Epstein, 1987). Plantas de alimentación. La riqueza local y la abundancia, así como la distribución espacial y temporal de los papilionoideos están correlacionadas estrechamente con las plantas de alimentación larval (Shields et al., 1969; Gilbert, 1984) y su fenología. Algunas especies de mariposas se alimentan de una sola especie vegetal (monofagia), y otras utilizan una amplia variedad de plantas de alimentación (polifagia), sin embargo, la gran mayoría está confinada a un grupo de huéspedes taxonómica y químicamente relacionados (oligofagia), o que al menos contienen compuestos similares como aceites y glucósidos, pero ninguna especie de mariposa se alimenta indiscriminadamente de todas las plantas (Ehrlich y Raven, 1967; Shields et al., 1969; Gilbert y Singer, 1975; VaneWright, 1978; Scott, 1986). En ocasiones simplemente se catalogan como especialistas y generalistas (Shapiro, 1973 en Luis y Llorente, 1990; Novotny y Basset, 2005).

De acuerdo con los resultados, es probable que al menos el $10 \%$ de la ropalocerofauna (14 especies) sea residente, pues en Cañón de Lobos está presente su especie huésped; la condición de residencia de estos papilionoideos se ve reforzada por su abundancia, especialmente en el caso de $E$. daira sidonia, H. hanno antibubastus, P. dina westwoodi, P. nise nelphe, C. l. lacinia, P. a. agarithe, L. marina y $P$. p. philea, que son especies abundantes y comunes en el área. Por ejemplo, E. daira sidonia es una especie que se encuentra tanto en ambientes templados como en los tropicales, aunque prefiere estos últimos en los que es más abundante; esta distribución amplia sugiere que es una especie generalista que utiliza gran variedad de recursos (Howe, 1975; DeVries, 1985, 1987; Scott, 1986), por lo que es capaz de ocupar un intervalo geográfico extenso. 
El resto de las especies residentes también es generalista, pues utiliza gran variedad de recursos alimenticios y tiene distribución amplia; Phoebis p . philea se distingue en este grupo pues se alimenta de varias especies, pero únicamente del género Cassia (Lamas, 1984; DeVries, 1985, 1987; Scott, 1986).

Para la mayor parte de los Papilionoidea de Cañón de Lobos al menos está presente el género de la planta de alimentación; la abundancia de algunas especies y muchos imagos recién emergidos indica que se reproducen en el área. Estas mariposas tienen huéspedes numerosos que incluyen varios géneros, lo cual sugiere que a pesar que en Cañón de Lobos no está presente la especie de alimentación citada en la literatura, las mariposas se alimentan de los recursos disponibles en la localidad, entre los cuales se encuentran otras especies del mismo género del huésped (Gilbert y Singer, 1975) (Cuadro 3). Incluso existe la posibilidad de que los papilionoideos se alimenten de huéspedes aún no conocidos, pues a diferencia de las zonas templadas, en los trópicos las mariposas utilizan a huéspedes de mayor diversidad de familias (Godfray et al., 1999), es el caso de B. p. polydamas y P. montezuma que se nutren exclusivamente de plantas de Aristolochia, mientras E. hegesia meridiania lo hace de Passiflora, géneros para los que no se ha reconocido la presencia de las especies en Cañón de Lobos; no obstante, es muy probable que estas mariposas se alimenten de ellas; o como E. arbela boisduvaliana que come plantas del género Cassia (Scott, 1986), del cual hay 5 especies disponibles en el área (Chavelas, 1972; Vázquez, 1974; Soria, 1986).

Se conoce que las larvas de algunas especies de Papilionoidea se alimentan de un solo huésped, como B. $b$. brevicornis, Eurema salome jamapa, C. miserabilis, Rekoa palegon, R. zebina, A. jada, O. ocrisia, S. bebrycia, M. clytie, Thisbe lycorias y C. h. hippodrome, papilionoideos que suman menos del $10 \%$ del total de las 120 especies analizadas. A menudo, las mariposas utilizan un espectro amplio de huéspedes, y una pequeña proporción de la comunidad tiene un huésped específico (Godfray et al., 1999). La especificidad se incrementa en los ambientes tropicales, pues sólo un tercio de estos herbívoros se alimenta exclusivamente de una especie de planta, aun cuando estén disponibles huéspedes congenéricos alternativos. En contraste, el porcentaje de especies que se alimenta de un género (48\%) o familia (58\%) es alto (Novotny y Basset, 2005). La mayoría de estas especies monófagas pertenecen a Lycaenidae, familia que se caracteriza por su notable especificidad al huésped, ya que un tercio de sus especies únicamente se nutre de una especie vegetal y el resto está restringida a un género o familia de plantas (Fiedler, 1996), como puede reconocerse en la lista al principio de este párrafo.
Bolboneura s. beatrix, M. c. cyananthe, C. rosita riobalsensis y $A$. t. texana son las más abundantes entre las especies para las que se registró la familia del huésped en el área de estudio; las familias completas de plantas referidas como huéspedes de estos papilionoideos revela que pueden ser generalistas o tal vez oligófagos (Kendall, 1964; Ehrlich y Raven, 1967; Beutelspacher, 1980; Scott, 1986; DeVries, 1987; Maza, 1987), y por consiguiente, residentes que pueden utilizar las especies de estas familias vegetales presentes en Cañón de Lobos (Chavelas, 1972; Vázquez, 1974; Soria, 1986).

Quizá en esta misma situación se encuentren algunos de los papilionoideos para los que no se registró la especie, género o familia de su huésped en Cañón de Lobos. En general, estas mariposas fueron menos abundantes, pues se ubicaron en las categorías frecuente, escasa o rara (Cuadro 1). Además, de acuerdo con los huéspedes citados en la literatura, la mayoría se alimenta de un género en particular, o de 2 o más géneros de la misma familia, por lo que podrían considerarse oligófagos ; tal vez es posible que en el área de estudio utilicen otros recursos que aún no se conocen, como en el caso de C. ehrenbergii y E. z. zela que fueron especies frecuentes. Debido a la especialización por su huésped (Cuadro 3), P. epidaus fenochionis, C. $n$. nimbice y $C$. $h$. hippodrome son las especies que tienen menor probabilidad de ser residentes en Cañón de Lobos. Sin embargo, para las 2 primeras basta con una población escasa del huésped, no detectada por los botánicos, para que su residencia sea justificada.

Por otra parte, Protographium epidaus fenochionis y $C$. $h$. hippodrome pueden ser especies escasas o raras en otros sitios de la vertiente del Pacífico con vegetación de selva baja caducifolia, subcaducifolia y mediana perennifolia (Luis et al., 1991; Vargas et al., 1992; Luna-Reyes et al., 2008). Catasticta $n$. nimbice principalmente se distribuye en zonas frías y templadas, aunque es una especie común en localidades más cálidas de Morelos como Tepoztlán (Maza, 1975) y El Chichinautzin (Valencia, 1999), ubicadas en el Eje Neovolcánico en un área próxima a Cañón de Lobos, por lo que es posible que se hayan desplazado hasta Cañón de Lobos, con natural vagilidad en busca de alimento.

De las 8 especies exclusivas encontradas en la SBC, todas excepto 3 : C. rawsoni, C. w. wellingi y J. e. nigrosuffusa, han sido registradas en extensas zonas tropicales del país (Llorente et al., 2006) que siempre coinciden con la distribución de la selva baja caducifolia. Sin embargo, es necesario hacer un análisis más detallado que permita definir con precisión la exclusividad de dichas especies.

Dado que la zona de estudio fue alterada por el trazo de una carretera en la década de 1980, será de interés conocer el efecto de ésta después de 2 décadas. 


\section{Agradecimientos}

A la Carrera de Biología de la Facultad de Estudios Superiores Zaragoza, UNAM. En especial a Francisco Javier Ramos Durón por su invaluable colaboración en la recolecta de ejemplares. Los proyectos de DGAPAUNAM PAPIIT IN203509 y PE 201507, y CONACYT 83237 fueron de gran apoyo para los 3 últimos autores en el desarrollo de este trabajo.

\section{Literatura citada}

Arteaga, G. L. 1991. Aspectos de la distribución y fenología de los Papilionoidea (Insecta: Lepidoptera) de la cañada de los Chorros del Varal, Municipio de Los Reyes, Michoacán. Tesis, Facultad de Biología, Universidad Michoacana de San Nicolás de Hidalgo, Morelia, Michoacán, México. 70 p.

Austin, G. T. 1978. Phenology and diversity of a butterfly population in southern Arizona. Journal of the Lepidopterists' Society 32:207-220.

Balcázar, L. M. 1993. Butterflies of Pedernales, Michoacán, Mexico, with notes on seasonality and faunistic affinities (Lepidoptera: Papilionoidea and Hesperoidea). Tropical Lepidoptera 4:93-105.

Beutelspacher, C. R. 1980. Mariposas diurnas del Valle de México, Ediciones Científicas. La Prensa Médica Mexicana, México, D. F. 134 p. + XVI lám.

Beutelspacher, C. R. 1982. Lepidópteros de Chamela, Jalisco, México I: Rhopalocera. Anales del Instituto de Biología, Universidad Nacional Autónoma de México, Serie Zoología 52:371-388.

Beutelspacher, C. R. 1984. Mariposas de México I. Introducción y generalidades. Superfamilia Papilionoidea. Familia Papilionidae. La Prensa Médica Mexicana, México, D. F. 128 p. + 20 lám.

Bizuet, F. Y., A. Luis y J. Llorente B. 2001. Mariposas del Parque Nacional El Chico, Hidalgo y sus relaciones biogeográficas con cinco zonas aledañas al Valle de México,México(Lepidoptera:Papilionoidea).Sociedad Hispano-Luso-Americana de Lepidopterología, Revista de Lepidopterología 29:145-159.

Brown, K. S. 1979. Maximizing daily butterfly counts. Journal of the Lepidopterists' Society 26: 183-195.

Cam, E., J. Nichols, J. Sauer y J. Hines. 2002. On the estimation of species richness based on the accumulation of previously unrecord species. Ecography, Pattern and Diversity in Ecology 25:102-108.

Chavelas, P. J. 1972. Excursión al Cañón de Lobos, Mor. In Guías botánicas de excursiones en México. Sociedad Botánica de México, México, D. F. p. 45-57.
Clench, H. K. 1979. How to make regional list of butterflies: some thoughts. Journal of the Lepidopterists' Society 33:216-231.

Clench, H. K. 1981. New Callophrys (Lycaenidae) from North and Middle America. Bulletin of The Allyn Museum 64:1-31.

Comstock, J. A. y L. Vázquez. 1961. Estudio de los ciclos biológicos en lepidópteros mexicanos. Anales del Instituto de Biología, Universidad Nacional Autónoma de México 31:349-448.

Corona, N. E. V. 1967. Introducción al estudio de la flora de los alrededores de Cuernavaca, Morelos, México. Tesis, Facultad de Ciencias, Universidad Nacional Autónoma de México, México, D. F. 67 p.

Courtney, S.P. 1986. The ecology of Pierid Butterflies: dynamics and interactions. Advances in ecological Research 15:51-131.

DeVries, P. J. 1985. Host plant records and natural history notes on Costa Rica butterflies (Papilionidae, Pieridae \& Nymphalidae). Journal of the Lepidopterists' Society 24: 290-332.

DeVries, P. J. 1987. The butterflies of Costa Rica and their natural history. Papilionidae, Pieridae, Nymphalidae. Princeton University Press, New Jersey. 327 p.

DeVries, P. J. 1997. The butterflies of Costa Rica and their natural history. II Riodinidae. Princeton University Press, New Jersey. 288 p.

Ehrlich, P. R. 1984. The structure and dynamics of butterfly populations. In The biology of butterflies, R. I. VaneWright y P. R. Ackery (eds.). Academic, London. p. 25-40.

Ehrlich, P. R. y H. Ehrlich. 1961. How to know the butterflies. William C. Brown, Dubuque, Iowa. 262 p.

Ehrlich, P. R. y P. H. Raven. 1965. Butterflies and plants: a study in coevolution. Evolution 18:586-608.

Ehrlich, P. R. y P. H. Raven. 1967. Butterflies and plants. Scientific American 216:104-113.

Emmel, J. F., O. Shields y D. E. Breedlove. 1970. Larval food plant records for Northamerican Rhopalocera. Journal of Research on the Lepidoptera 9:233-242.

Emmel, T. C. y J. F. Emmel. 1973. The butterflies of southern California. Natural History Museum of Los Angeles County, Science Series 26. 148 p.

ENSO. 2006. El Niño southern oscillation. Earth System Research Laboratory, Physical Sciences Division, National Oceanic and Atmospheric Administration, Department of Commerce, United States. http//www. cdc.noaa.gov; 01.IX.2006.

Ferris, C. D. 1985. A new subspecies of Apodemia hypoglauca (Godman \& Salvin) from the Yucatan Peninsula (Lycaenidae: Riodinidae). Bulletin of The Allyn Museum 94:1-7. 
Fiedler, K. 1996. Host-plant relationships of lycaenid butterflies: large-scale patterns, interactions with plant chemistry, and mutualism with ants. Entomologia Experimentalis et Applicata 80:259-267.

Flores, V.O.y P. Gerez. 1994. Biodiversidad y conservación en México: vertebrados, vegetación y uso del suelo. Facultad de Ciencias, Universidad Nacional Autónoma de México /Comisión Nacional para el Conocimiento y Uso de la Biodiversidad, México, D. F. 439 p.

FURN. 2006. Host Plants Index. Finnish University and Research Network. http://www.funet.fi/pub/sci/bio/ life/warp/food-plants-a.html; 01.III.2008.

Gilbert, L. E. 1984. The biology of butterfly communities. In The biology of butterflies, R. I. Vane-Wright y P. R. Ackery (eds.). Academic, London. p. 41-64.

Gilbert, L. E. y M. C. Singer. 1975. Butterfly Ecology. Annual Review of Ecological Systems 6:365-397.

Godfray, H. C. J., O. T. Lewis y J. Memmott. 1999. Studying insect diversity in the tropics. Philosophical Transactions of the Royal Society of London Series B 354:1811-1824.

Godman, F. D. e I. O. Salvin. 1869-1901. Biologia Centrali-Americana, vols. I, II, III. Zoología, Insecta. Lepidoptera Rhopalocera. Bernard Quaritch, Dulau, London. 782 p.

Howe, W. H. 1975. The Butterflies of North America. Doubleday, Garden City, New York. 633 p.

Hoffmann, C. C. 1940. Catálogo sistemático y zoogeográfico de los lepidópteros mexicanos. Primera parte. Papilionoidea. Anales del Instituto de Biología, Universidad Nacional Autónoma de México 11:639-739.

Jenkins, D. W. 1983. Neotropical Nymphalidae I. Revision of Hamadryas. Bulletin of The Allyn Museum 81:1-146.

Jenkins, D. W. 1984. Neotropical Nymphalidae II. Revision of Myscelia. Bulletin of The Allyn Museum 87:1-64.

Jiménez -Valverde, A. y J. Hortal. 2003. Las curvas de acumulación de especies y la necesidad de evaluar la calidad de los inventarios biológicos. Revista Ibérica de Aracnología 8:151-161.

Kendall, R. O. 1964. Larval food plants for twenty six species of Rhopalocera (Papilionoidea) from Texas. Journal of the Lepidopterists' Society 18:129-157.

Kendall, R. O. 1976. Larval fooplants and life history for some metalmarks (Lepidoptera: Riodinidae) from Mexico and Texas. Bulletin of the Allyn Museum 32:1-12.

Kendall, R. O. y W. W. McGuire. 1984. Some new and rare records of Lepidoptera found in Texas. Bulletin of the Allyn Museum 86:1-50.

Klots, A. 1951. A field guide to the butterflies. Houghton
Mifflin, Boston, Massachusetts. 349 p.

Krebs, C. J. 1985. Ecología. Estudio de la distribución y abundancia. Harla, México, D. F. 753 p.

Lamas, G. 1984. Los Papilionoidea (Lepidoptera) de la zona Reservada de Tambopata, Madre de Dios, Perú. I Papilionidae, Pieridae y Nymphalidae (en parte). Revista Peruana de Entomología 27:59-73.

Lara, A. M. 1999. Riqueza de especies y abundancia relativa de la superfamilia Papilionoidea(Lepidoptera) en un bosque de Quercus, en Huitzilac, Morelos. Tesis, Facultad de Estudios Superiores Zaragoza, Universidad Nacional Autónoma de México, México, D. F. 69 p.

Llorente, B. J., A. Luis, I. Vargas F. y J. Soberón M. 1996. Papilionoidea (Lepidoptera). In Biodiversidad, taxonomía y biogeografía de artrópodos de México: hacia una síntesis de su conocimiento, J. Llorente, A. García y E. González (comps.). Comisión Nacional para el Conocimiento y Uso de la Biodiversidad / Facultad de Ciencias, Universidad Nacional Autónoma de México, México, D. F. p. 531-348.

Llorente, B. J., L. Oñate, A. Luis M. e I. Vargas F. 1997. Papilionidae y Pieridae de México: Distribución geográfica e Ilustración. Comisión Nacional para el Conocimiento y Uso de la Biodiversidad / Facultad de Ciencias, Universidad Nacional Autónoma de México, México, D. F. 227 p.

Llorente, B. J., A. Luis M. e I. Vargas F. 2006. Apéndice general de Papilionoidea: Lista sistemática, distribución estatal y provincias biogeográficas. In Componentes bióticos principales de la entomofauna mexicana, J. J. Morrone y J. Llorente (eds.). Las Prensas de Ciencias, Facultad de Ciencias, Universidad Nacional Autónoma de México, México, D. F. p. 945-1009.

Luis, M. A. y J. Llorente. 1990. Mariposas del Valle de México: Introducción e historia I. Distribución local y estacional de los Papilionoidea de la cañada de los Dínamos, Magdalena Contreras, D. F., México. Folia Entomológica Mexicana 78:95-198.

Luis, M. A. y J. Llorente. 1993. Mariposas. In Historia natural del parque ecológico estatal Omiltemi, Chilpancingo, Guerrero, México, I. Luna V. y J. Llorente B. (eds.). Comisión Nacional para el Conocimiento y Uso de la Biodiversidad / Facultad de Ciencias, Universidad Nacional Autónoma de México, México, D. F. p. 307-385.

Luis, M. A., I. Vargas y J. Llorente. 1991. Lepidopterofauna de Oaxaca I: Distribución y fenología de los Papilionoidea de la sierra de Juárez. Publicaciones especiales del Museo de Zoología 3, Facultad de Ciencias, Universidad Nacional Autónoma de México, México, D. F. 118 p. 
Luis, M. A., J. Llorente e I. Vargas. 2003a. Nymphalidae de México I (Danainae, Apaturinae, Biblidinae y Heliconiinae): distribución geográfica e ilustración. Comisión Nacional para el Conocimiento y Uso de la Biodiversidad / Universidad Nacional Autónoma de México, México, D. F. 249 p.

Luis, M. A., J. Llorente, I. Vargas y A. Warren. 2003b. Biodiversity and biogeography of mexican butterflies (Lepidoptera: Papilionoidea and Hesperoidea). Proceedings of the Entomological Society of Washington 105:209-224.

Luna-Reyes, M. 2007a. Estudio faunístico sobre Papilionoidea (Lepidoptera) en Cañón de Lobos, Yautepec, Morelos. Tesis, Maestría Facultad de Ciencias, Universidad Nacional Autónoma de México, México, D. F. 102 p.

Luna-Reyes, M. 2007b. Distribución y estacionalidad de las mariposas de la sierra Nevada, México (Lepidoptera: Papilionoidea). In Biodiversidad de la faja volcánica transmexicana, I. Luna, J. J. Morrone y D. Espinosa (eds.), Las Prensas de Ciencias, Facultad de Ciencias, Universidad Nacional Autónoma de México, México, D. F. p. 341-375.

Luna-Reyes, M. y J. Llorente. 2004. Papilionoidea (Lepidoptera: Rhopalocera) de la sierra Nevada, México. Acta Zoológica Mexicana 20:79-102.

Luna-Reyes, M., J. Llorente y A. Luis. 2008. Papilionoidea de la sierra de Huautla, Morelos y Puebla, México (Insecta: Lepidoptera). Revista de Biología Tropical (International Journal of Tropical Biology and Conservation) 56:1677-1716.

Magaña, R. V., J. L. Pérez, C. Conde, C. Gay y S. Medina. 2006. El fenómeno de El Niño y la oscilación del sur (ENOS) y sus impactos en México. Departamento de Meteorología General, Centro de Ciencias de la Atmósfera, Universidad Nacional Autónoma de México. http://www.atmosfera.unam.mx/cambio/nino. htm; 15.VIII.2008.

McAlpine, W. S. 1971. A revision of the butterfly genus Calephelis (Riodininae). Journal of Research on the Lepidoptera 10:1-125.

Masters, J. H. 1970. Distributional notes on the genus Mestra (Nymphalidae) in North America. Journal of the Lepidopterists' Society 24:203-208.

Maza, E. R. de la. 1975. Notas sobre lepidópteros de Rancho Viejo y Tepoztlán, Morelos, México. Primera parte: Papilionoidea. Revista de la Sociedad Mexicana de Lepidopterología 1:42-61.

Maza, E. R. de la., A. White L. y A. Ojeda C. 1995a. La horofauna hidrófila de la Cañada de la Toma, Tilzapotla, Morelos, México. (LepidopteraRhopalocera). Revista de la Sociedad Mexicana de
Lepidopterología 15:1-38.

Maza, E. R. de la, A. White L. y R. de la Maza R. 1995b. Exploración de factores compensatorios que permiten el refugio de ropalocerofauna hidrófila en cinco cañadas de clima subhúmedo en Morelos, México. Revista de la Sociedad Mexicana de Lepidopterología 16:1-63.

Maza, R. R. de la y R. Turrent. 1977. Un nuevo Calephelis de la cuenca superior del río Balsas, México (Riodinidae). Revista de la Sociedad Mexicana de Lepidopterología 3:85-90.

Maza, R. R. de la. 1987. Mariposas mexicanas. Guía para su colecta y determinación. Fondo de Cultura Económica, México, D. F. 302 p.

Maza, R. R. de la y A. White. 1990. Rhopalocera de la Huasteca potosina, su distribución, composición, origen y evolución. Revista de la Sociedad Mexicana de Lepidopterología 13:31-87.

Medellín, H. F. 1985. Mariposas diurnas del suborden Rhopalocera (Lepidoptera) de la localidad de "El Limón", Municipio de Tepalcingo Morelos. Tesis, Escuela de Ciencias Biológicas, Universidad Autónoma del Estado de Morelos, Cuernavaca. 108 p.

Miller, L. D. 1978. Revision of the Euptychiini (Satyridae). Bulletin of the Allyn Museum 3: 1-12.

Miller, L. D. y J. Y. Miller. 1988. A new Euptychia species from nortwestern Mexico (Satyridae). Journal of the Lepidopterists' Society 42:276-280.

Miranda, F. y E. Hernández X. 1963. Los tipos de vegetación en México y su clasificación. Boletín de la Sociedad Botánica de México 28:29-179.

Moore, N. W. 1975. Butterfly transects in a linear habitat 1964-1973. Entomologist's Gazette 26:71-78.

Moreno, C. E. y G. Halffter. 2001. On the measure of sampling effort used in species accumulation curves. Journal of Applied Ecology 38:487-490.

Morón, R. M. y R. A. Terrón. 1984. Distribución altitudinal y estacional de los insectos necrófilos en la sierra Norte de Hidalgo, México. Acta Zoológica Mexicana 3:147.

Neck, R. W. 1973. Foodplant ecology of the butterfly Chlosyne lacinia (Geyer) (Nymphalidae) I. Larval foodplants. Journal of the Lepidopterists' Society 27:22-33.

Nicolay, S. S. 1976. A review of the Hübnerian genera Panthiades and Cycnus (Lycaenidae: Eumaeini). Bulletin of the Allyn Museum 35:1-30.

Novotny, V. y Y. Basset. 2005. Host specificity of insect herbivores in tropical forests. Proceedings of the Royal Society of London, Series B 272:1083-1090.

Owen, D. F. 1971. Tropical butterflies. Oxford University Press, London. 215 p.

Pollard, E. 1977. A method for assessing changes in the 
abundance of butterflies. Biological Conservation 12:115-134.

Pyle, R. M. y A. A. Knopf. 1981. The Audubon Society field guide to North American butterflies. Chanticleer Press, New York. 916 p.

Rhoades, D. F. 1983. Herbivore population dynamics and plant chemistry. In Variable plants and herbivores in natural and managed systems, R. F. Denno y M. S. McClure (eds.). Academic, New York. p. 55-68.

Riley, N. D. 1975. A field guide to the butterflies of the West Indies. Collins, London. 224 p.

Rodríguez, N. S. 1982. Mariposas del suborden Rhopalocera (Lepidoptera) de Acatlán de Juárez, Jalisco y alrededores. Tesis, Facultad de Ciencias, Universidad Nacional Autónoma de México, México, D.F. 206 p.

Ross, E. S. 1964a. Life history studies on Mexican butterflies I. Notes on the early stages of four Papilionids from Catemaco, Veracruz. Journal of Research on the Lepidoptera 3:9-18.

Ross, E. S. 1964b. Life history studies on Mexican butterflies III. Nine Rhopalocera (Papilinidae, Nymphalidae, Lycaenidae) fron Ocotal Chico, Veracruz. Journal of Research on the Lepidoptera 3:207-229.

Rzedowski, J. 1978. Vegetación de México. Limusa, México. D. F. 432 p.

Rzedowski, J. 1992. El endemismo en la flora fanerogámica de México. In La diversidad biológica de Iberoamérica I, G. Halffter (comp.), Acta Botanica Mexicana, volumen especial: 337-359.

Rzedowski, G. C. y J. Rzedowski. 2001. Flora fanerogámica del Valle de México. Instituto de Ecología / Comisión Nacional para el Conocimiento y Uso de la Biodiversidad, Pátzcuaro, Michoacán. 1406 p.

Scott, J. A. 1986. The butterflies of North America. A natural history and field guide. Standford University Press, California. 583 p.

Scott, J. A. y M. E. Epstein. 1987. Factors affecting phenology in a temperate insect community. The American Midland Naturalist 117:103-118.

Scriber, J. M. y P. P. Feeny. 1976. New food-plant and oviposition records for Battus philenor (Papilionidae). Journal of the Lepidopterists' Society 30:70-71.

Serrano, F. y M. E. Serrano. 1972. Las mariposas de El Salvador, Primeraparte.Papilionidae, Comunicaciones, Instituto de Ciencias Naturales, Universidad de El Salvador 1:44-80.

Seitz, A. (ed.). 1912-1924. Die Gross- Schmetterlinge der Erde. Alfred Kernen, Stuttgart.

Shapiro, A. M. 1974. Butterflies and skippers of New York State. Search Agriculture, Entomology 4:1-60.

Shapiro, A. M. 1975. The temporal component of butterfly species. Diversity. In Ecology and evolution of communities, M. L. Cody y J. M. Diamond (eds.). The Belknap Press of Harvard University Press, Cambridge, Massachusetts p. 181-195.

Shields, O., J. F. Emmel y D. E. Breedlove. 1969. Butterfly larval foodplant records and a procedure for reporting foodplants. Journal of the Lepidopterists' Society 8:21-36.

Silva, L. P. y M. Ibarra. 2003. Lepidópteros diurnos de la sierra de Huautla, Morelos. Entomología Mexicana 2:230-235.

Smith, R. L. 1980. Ecology and field biology, tercera edición, Harper \& Row, New York. 835 p.

Soberón J. y J. Llorente. 1993. The use of species accumulation functions for the predictions of species richness. Conservation Biology 7:480-488.

Soria, R. G. 1986. Flora de Morelos. Descripción de especies vegetales de la selva baja caducifolia del Cañón de Lobos, Municipio de Yautepec. Serie Ciencias Naturales y de la Salud. Universidad Autónoma del Estado de Morelos, Cuernavaca. 206 p.

SPP (Secretaría de Programación y Presupuesto). 1981. Síntesis geográfica de Morelos. Coordinación General de los Servicios Nacionales de Estadística, Geografía e Informática, Secretaría de Programación y Presupuesto, México, D. F. 110 p.

Trejo, I. 1996. Características del medio físico de la selva baja caducifolia en México. Investigaciones Geográficas Boletín, número especial 4:95-110.

Tietz, H. M. 1972. An index to the described life histories, early ages and hosts of the macrolepidoptera of the continental United States and Canada. Allyn Museum of Entomology, Sarasota, Florida. 1041 p.

Tyler, H. 1975. The swallowtail butterflies of North America. Naturegraph, Healdsburg, California. 192 p.

Valencia, G. M. 1999. Listado faunístico de los Papilionoidea (Lepidoptera: Rhopalocera) del derrame lávico del volcán Chichinautzin, estado de Morelos. Tesis, Facultad de Ciencias, Universidad Nacional Autónoma de México, México, D. F. 46 p.

Vane-Wright, R. I. 1978. Ecological and behavioral origins of diversity in butterflies. In Diversity of insect faunas, L. A. Mound y N. Waloff (eds.). Blackwell Scientific, Oxford. p. 56-69.

Vargas, I., J. Llorente y A. Luis. 1992. Listado lepidopterofaunístico de la Sierra de Atoyac de Álvarez en el estado de Guerrero: notas acerca de su distribución local y estacional (Rhopalocera: Papilionoidea). Folia Entomológica Mexicana 86:41-178.

Vargas, I., J. Llorente y A. Luis. 1999. Distribución de los Papilionoidea (Lepidoptera: Rhopalocera) de la sierra de Manantlán (250-1,650 m) en los estados de Jalisco 
y Colima. Publicaciones Especiales 11, Museo de Zoología, Facultad de Ciencias, Universidad Nacional Autónoma de México, México D. F. 53 p.

Vázquez, G. L. y H. Pérez. 1962. Observaciones sobre la biología de Baronia brevicornis Salv. (Lepidoptera: Papilionidae-Baroniinae). Anales del Instituto de Biología, Universidad Nacional Autónoma de México, Serie Zoología 32:295-311.

Vázquez, S. J. 1974. Contribución al estudio de las plantas del estado de Morelos (México). Catálogo de las plantas contenidas en el Herbario "L' Amagatall". Revista
Ciencia, Academia Mexicana de Ciencias 29:1-138.

Vences, B. M. O. 2004. Lista lepidopterofaunística del estadodeMorelos(Insecta:Lepidoptera:Papilionoidea). Tesis, Facultad de Ciencias, Universidad Nacional Autónoma de México, México, D. F. 51 p.

Vidal, R. 1980. Algunas relaciones clima-cultivos en el estado de Morelos. Publicaciones del Instituto de Geografía, Universidad Nacional Autónoma de México, México, D. F. 95 p.

Wolda, H. 1988. Insect seasonality: Why? Annual Review of Ecology and Systematics 19:1-18.

Apéndice 1. Composición faunística de Papilionoidea en Cañón de Lobos. En la lista que se presenta a continuación las especies tienen la nomenclatura y el orden filogenético propuesto por Llorente et al. (2006); Lycaenidae se refiere a las familias Lycaenidae y Riodinidae reconocidas por estos autores.

\section{PAPILIONIDAE \\ Baroniinae \\ 1. Baronia brevicornis brevicornis Salvin, 1893 Papilioninae \\ 2. Protographium epidaus fenochionis (Salvin et Godman, 1868) \\ 3. Mimoides thymbraeus aconophos (Gray, [1853]) \\ 4. Battus philenor philenor (Linnaeus, 1771) \\ 5. Battus polydamas polydamas (Linnaeus, 1758) \\ 6. Parides erithalion trichopus (Rothschild et Jordan, 1906) \\ 7. Parides montezuma (Westwood, 1842) \\ 8. $\quad$ Parides photinus (Doubleday, 1844) \\ 9. Heraclides cresphontes (Cramer, 1777) \\ 10. Heraclides rogeri pharnaces (Doubleday, 1846) \\ 11. Heraclides thoas autocles (Rothschild et Jordan, 1906) \\ 12. Papilio polyxenes asterius Stoll, 1782 \\ 13. Pterourus garamas garamas (Geyer, [1829]) \\ 14. Pterourus multicaudata multicaudata (W.F. Kirby, 1884)}

\section{PIERIDAE}

Coliadinae

15. Zerene cesonia cesonia (Stoll, 1790)

16. Anteos clorinde (Godart, [1824])

17. Anteos maerula (Fabricius, 1775)

18. Phoebis agarithe agarithe (Boisduval, 1836)

19. Phoebis argante ssp. $\mathrm{n}$.

20. Phoebis neocypris virgo (Butler, 1870)

21. Phoebis philea philea (Linnaeus, 1763)

22. Phoebis sennae marcellina (Cramer, 1777)

23. Rhabdodryas trite ssp. $\mathrm{n}$.

24. Abaeis nicippe (Cramer, 1779)

25. Pyrisitia dina westwoodi (Boisduval, 1836)

26. Pyrisitia nise nelphe (R. Felder, 1869)

27. Pyrisitia proterpia (Fabricius, 1775)

28. Eurema arbela boisduvaliana (C. Felder et R.
Felder, 1865)

29. Eurema daira sidonia (R. Felder, 1869)

30. Eurema mexicana mexicana (Boisduval, 1836)

31. Eurema salome jamapa (Reakirt, 1866)

32. Nathalis iole Boisduval, 1836 Pierinae

33. Hesperocharis costaricensis pasion (Reakirt, [1867])

34. Catasticta nimbice nimbice (Boisduval, 1836)

35. Glutophrissa drusilla tenuis Lamas, 1981

36. Leptophobia aripa elodia (Boisduval, 1836)

37. Ascia monuste monuste (Linnaeus, 1764)

38. Ganyra josephina josepha (Salvin et Godman, 1868)

\section{LYCAENIDAE}

Theclinae

39. Rekoa palegon (Cramer, 1780)

40. Rekoa zebina Hewitson

41. Arawacus jada (Hewitson, 1867)

42. Contrafacia bassania (Hewitson, 1868)

43. Satyrium polingi (Barnes et Benjamin, 1926)

44. Ocaria ocrisia (Hewitson, 1868)

45. Chlorostrymon simaethis (Drury, 1773)

46. Cyanophrys herodotus (Fabricius, 1793)

47. Cyanophrys miserabilis (Clench, 1946)

48. Allosmaitia strophius (Godart, [1824])

49. Calycopis isobeon (Butler et H. Druce, 1872)

50. Strymon bebrycia (Hewitson, 1868)

51. Strymon yojoa (Reakirt, [1867])

53. Strymon istapa (Reakirt, [1867])

52. Strymon bazochii (Godart, [1824])

54. Strymon sp.

55. Ministrymon clytie (W.H. Edwards, 1877)

56. Panthiades bathildis (C. Felder et R. Felder, 1865)

Polyommatinae

57. Leptotes cassius cassidula (Boisduval, 1870)

58. Leptotes marina (Reakirt, 1868)

59. Zizula cyna (W.H. Edwards, 1881) 
60. Cupido comyntas (Godart, [1824])

61. Celastrina argiolus gozora (Boisduval, 1870)

62. Hemiargus hanno antibubastus Hübner, [1818]

63. Echinargus isola (Reakirt, [1867]) Riodininae

64. Calephelis perditalis perditalis Barnes et $\mathrm{Mc}$ Dunnough, 1918

65. Calephelis rawsoni McAlpine, 1939

66. Calephelis wellingi wellingi McAlpine, 1971

67. Calephelis sp. 1

68. Calephelis sp. 2

69. Calephelis sp. 3

70. Calephelis sp. 4

71. Calephelis sp. 5

72. Caria ino ino Godman et Salvin, 1886

73. Caria stillaticia Dyar, 1912

74. Baeotis zonata zonata R. Felder, 1869

75. Lasaia sula sula Staudinger, 1888

76. Lasaia maria maria Clench, 1972

77. Lasaia sp.

78. Melanis cephise acroeluca (R. Felder, 1869)

79. Calydna sturnula (Geyer, 1837)

80. Emesis mandana furor Butler et H. Druce, 1872

81. Emesis tegula Godman et Salvin, 1886

82. Emesis poeas Godman, 1901

83. Emesis tenedia C. Felder et R. Felder, 1861

84. Emesis zela zela Butler, 1870

85. Emesis emesia emesia (Hewitson, 1867)

86. Emesis sp. 1

87. Emesis sp. 2

88. Emesis sp. 3

89. Apodemia hypoglauca hypoglauca (Godman et Salvin, 1878)

90. Apodemia walkeri Godman et Salvin, 1886

91. Apodemia sp.

92. Thisbe lycorias (Hewitson, [1853])

\section{NYMPHALIDAE}

Libytheinae

93. Libytheana carinenta mexicana Michener, 1943 Danainae

94. Danaus eresimus montezuma Talbot, 1943

95. Danaus gilippus thersippus (H.W. Bates, 1863)

96. Danaus plexippus plexippus (Linnaeus, 1758) Morphiinae

97. Morpho polyphemus polyphemus Westwood, [1850]

98. Opsiphanes boisduvallii Doubleday, [1849] Satyrinae

99. Cissia similis (Butler, 1867)

100. Cissia themis (Butler, 1867)

101. Cyllopsis pyracmon pyracmon (Butler, 1867)

102. Euptychia fetna Butler, 1870

103. Hermeuptychia hermes (Fabricius, 1775)
104. Megisto rubricata anabelae L.D. Miller, 1976

105. Pindis squamistriga R. Felder, 1869

Charaxinae

106. Anaea troglodyta aidea (Guérin-Méneville, [1844]) Biblidinae

107. Marpesia petreus ssp. n.

108. Mestra dorcas amymone (Ménétriès, 1857)

109. Myscelia cyananthe cyananthe $\mathrm{C}$. Felder et R. Felder, 1867

110. Myscelia ethusa ethusa (Doyère, [1840])

111. Hamadryas atlantis lelaps (Godman et Salvin, 1883)

112. Hamadryas februa ferentina (Godart, [1824])

113. Bolboneura sylphis beatrix R. G. Maza, 1985

114. Epiphile adrasta adrasta Hewitson, 1861

115. Cyclogramma bacchis (Doubleday, 1849)

116. Adelpha iphicleola iphicleola (H.W. Bates, 1864)

Apaturinae

117. Asterocampa idyja argus (H. W. Bates, 1864)

118. Doxocopa laure laure (Drury, 1773)

Nymphalinae

119. Smyrna blomfildia datis Fruhstorfer, 1908

120. Nymphalis antiopa antiopa (Linnaeus, 1758)

121. Anartia fatima fatima (Fabricius, 1793)

122. Junonia coenia Hübner, [1822]

123. Junonia evarete nigrosuffusa Barnes et McDunnough, 1916

124. Siproeta epaphus epaphus (Latreille, [1813])

125. Siproeta stelenes biplagiata (Fruhstorfer, 1907)

126. Chlosyne ehrenbergii (Geyer, [1833])

127. Chlosyne hippodrome hippodrome (Geyer, 1837)

128. Chlosyne janais janais (Drury, 1782)

129. Chlosyne lacinia lacinia (Geyer, 1837)

130. Chlosyne marina marina (Geyer, 1837)

131. Chlosyne rosita riobalsensis Bauer, 1961

132. Chlosyne theona (Ménétriés, 1855)

133. Chlosyne sp.

134. Microtia elva elva H.W. Bates, 1864

135. Texola elada elada (Hewitson, 1868)

136. Anthanassa nebulosa alexon (Godman et Salvin, 1889)

137. Anthanassa texana texana (W.H. Edwards, 1863)

138. Phyciodes pallescens (R. Felder, 1869)

Heliconiinae

139. Euptoieta claudia daunius (Herbst, 1798)

140. Euptoieta hegesia meridiania Stichel, 1938

141. Agraulis vanillae incarnata (Riley, 1926)

142. Dione juno huascuma (Reakirt, 1866)

143. Dione moneta poeyii Butler, 1873

144. Dryas iulia moderata (Riley, 1926)

145. Heliconius charithonia vazquezae W.P.

Comstock et F.M. Brown, 1950 